\title{
AN AXIOMATIC DUALITY FRAMEWORK FOR THE THETA BODY AND RELATED CONVEX CORNERS
}

\author{
MARCEL K. DE CARLI SILVA AND LEVENT TUNÇEL
}

\begin{abstract}
Lovász theta function and the related theta body of graphs have been in the center of the intersection of four research areas: combinatorial optimization, graph theory, information theory, and semidefinite optimization. In this paper, utilizing a modern convex optimization viewpoint, we provide a set of minimal conditions (axioms) under which certain key, desired properties are generalized, including the main equivalent characterizations of the theta function, the theta body of graphs, and the corresponding antiblocking duality relations. Our framework describes several semidefinite and polyhedral relaxations of the stable set polytope of a graph as generalized theta bodies. As a by-product of our approach, we introduce the notion of "Schur Lifting" of cones which is dual to PSD Lifting (more commonly used in SDP relaxations of combinatorial optimization problems) in our axiomatic generalization. We also generalize the notion of complements of graphs to diagonally scaling-invariant polyhedral cones. Finally, we provide a weighted generalization of the copositive formulation of the fractional chromatic number by Dukanovic and Rendl.
\end{abstract}

\section{INTRODUCTION}

The Lovász theta function is one of the most elegant highlights in combinatorial and semidefinite optimization. First introduced in the seminal paper by Lovász [32] to solve a problem in information theory, the theta function was further developed in the 1980's along with applications of the ellipsoid method [22, 23, leading to the definition of the object known as the theta body of a graph as a semidefinite relaxation of its stable set polytope. This relaxation is tight for perfect graphs, and it leads to the only known (strongly) polynomial algorithm for finding optimal stable sets and colorings in such graphs. Since then, the theory surrounding the Lovász theta function has been further extended [34, 16, 37, 25, and it has been used in the design of approximation algorithms [28, 29, 2, 11, in complexity theory [46, 15, 5, 4, in information theory [38, 44, 12, and in extremal geometry [3.

For a graph $G=(V, E)$, the theta body of $G$ may be defined as the set

$$
\operatorname{TH}(G)=\left\{x \in \mathbb{R}^{V}: \exists X \in \mathbb{S}^{V}, X_{i i}=x_{i} \forall i \in V, X_{i j}=0 \forall i j \in E,\left[\begin{array}{cc}
1 & x^{\top} \\
x & X
\end{array}\right] \in \mathbb{S}_{+}^{\{0\} \cup V}\right\},
$$

where $\mathbb{S}^{V}$ denotes the set of $V \times V$ symmetric matrices and $\mathbb{S}_{+}^{\{0\} \cup V}$ is the set of symmetric positive semidefinite matrices on the index set $\{0\} \cup V$; we assume that 0 is not an element of $V$. The stable set polytope of $G$, denoted by $\operatorname{STAB}(G)$, is defined as the convex hull of incidence vectors of stable sets of $G$; a set $S \subseteq V$ is stable in $G$ if no edge of $G$ joins two elements of $S$. It is not hard to check that, if $x$ is the incidence vector of a stable set of $G$, then $X:=x x^{\top}$ satisfies the constraints on the RHS of (1.1), so $x \in \operatorname{TH}(G)$. Thus, the theta body is a relaxation of $\operatorname{STAB}(G)$, and the theta function

$$
\vartheta(G)=\max \left\{\sum_{i \in V} x_{i}: x \in \mathrm{TH}(G)\right\}
$$

is an upper bound for the size of a largest stable set in $G$. It is also convenient to define a weighted version $\vartheta(G ; w)$ of the theta function, by multiplying $x_{i}$ in the objective function of (1.2) by some weight $w_{i} \geq 0$ for each $i \in V$.

Date: August 3, 2018.

Part of the results in this paper appeared in the $\mathrm{PhD}$ thesis of the first author.

Research of the first author was supported in part by a Sinclair Scholarship, a Tutte Scholarship, Discovery Grants from NSERC, and by ONR research grant N00014-12-10049, while at the Department of Combinatorics and Optimization, University of Waterloo, and by grants 2013/20740-9 and 2013/03447-6, São Paulo Research Foundation (FAPESP), while at the Institute of Mathematics and Statistics, University of São Paulo.

Research of the second author was supported in part by a research grant from University of Waterloo, Discovery Grants from NSERC and by ONR research grant N00014-12-10049. 
Part of the broad applicability of the theta function owes to its multitude of equivalent formulations, which led Goemans to the conclusion that "it seems all paths lead to $\vartheta$ !" [20]. For instance, the eigenvalue formulation

$$
\vartheta(G)=\min \left\{\lambda_{\max }\left(A+\mathbb{1} \mathbb{1}^{\top}\right): A \in \mathbb{S}^{V}, A_{i j} \neq 0 \Longrightarrow i j \in E\right\},
$$

where $\lambda_{\max }$ extracts the largest eigenvalue and $\mathbb{1}$ is the vector of all-ones, is central for the approximate (vector) coloring algorithm of Karger, Motwani and Sudan [28]. Similarly, the non-convex formulation

$$
\vartheta(G)=\max \left\{\lambda_{\max }(B): B \in \mathbb{S}_{+}^{V}, B_{i i}=1 \forall i \in V, B_{i j}=0 \forall i j \in E\right\}
$$

essentially says that $\vartheta(G)$ is the best lower bound for the chromatic number of $G$ from a family of bounds due to Hoffman [26]; the chromatic number of $G$ is the minimum size of a partition of $V$ into stable sets of $G$. The description (1.1) itself is also especially well suited for proving that all (nontrivial) facets of $\operatorname{TH}(G)$ are determined by clique inequalities (see, e.g., [43, Theorem 67.13]), i.e., inequalities of the form $\sum_{i \in K} x_{i} \leq 1$ for some clique $K$ of $G$; a set $K \subseteq V$ is a clique of $G$ if every pair of elements of $K$ is an edge of $G$.

Most of these alternative formulations also apply to some classical variants of $\vartheta$, such as the functions $\vartheta^{\prime}$ and $\vartheta^{+}$defined as in (1.2) over corresponding variants of the theta body, denoted by $\mathrm{TH}^{\prime}(G)$ and $\mathrm{TH}^{+}(G)$, respectively (we define them in Section [2); see [39, 42, 45]. Many of such wealth of interesting characterizations arguably come from Semidefinite Programming (SDP) Strong Duality. A particularly illuminating manifestation of this duality is the identity (see, e.g., [43, Theorem 67.12])

$$
\operatorname{abl}(\operatorname{TH}(G))=\operatorname{TH}(\bar{G}),
$$

that is, the antiblocker of $\operatorname{TH}(G)$ is the theta body of $\bar{G}$, the complement of $G$. Antiblocking duality is the notion of duality most appropriate for a class of convex sets known as convex corners (defined in Section 2), which include all variants of theta bodies, as well as $\operatorname{STAB}(G)$. Similar instances of (1.4a) include

$$
\begin{aligned}
\operatorname{abl}\left(\mathrm{TH}^{\prime}(G)\right) & =\mathrm{TH}^{+}(\bar{G}), \\
\operatorname{abl}(\operatorname{STAB}(G)) & =\operatorname{QSTAB}(\bar{G}) ;
\end{aligned}
$$

here, $\operatorname{QSTAB}(G) \subseteq[0,1]^{V}$ is a classical polyhedral relaxation for $\operatorname{STAB}(G)$ determined by clique inequalities (we define it in Section 7). The relaxations in (1.4) are related by the following chain:

$$
\operatorname{STAB}(G) \subseteq \mathrm{TH}^{\prime}(G) \subseteq \mathrm{TH}(G) \subseteq \mathrm{TH}^{+}(G) \subseteq \operatorname{QSTAB}(G) \subseteq \operatorname{FRAC}(G) ;
$$

here, $\operatorname{FRAC}(G) \subseteq[0,1]^{V}$ is determined by edge inequalities, i.e., clique inequalities where the clique is a single edge (we define it in Section 4.2). The beautiful, striking relationships in (1.4) continue to manifest themselves in certain lift-and-project methods [1, 17, 31].

In this paper, we define a notion of generalized theta bodies and develop a duality theory that: (1) describes all sets in (1.5) as theta bodies, (2) extends many of the equivalent formulations for $\vartheta$ to the corresponding generalized theta functions, and (3) extends the antiblocker relations (1.4) to generalized theta bodies. We shall parameterize a generalized theta body, henceforth called just theta body, by two convex cones. One of them, which we denote by $\mathbb{A}$, shall encode the adjacency constraints $X_{i j}=0$ in (1.1), i.e., those constraints shall be replaced with " $X \in \mathbb{A}$ ". The other cone, which we denote by $\widehat{\mathbb{K}}$, will replace $\mathbb{S}_{+}^{\{0\} \cup V}$ in (1.1); it essentially constrains how $X$ and $x x^{\top}$ are related, so we may think of $\widehat{\mathbb{K}}$ as a cone that encodes quadratic relations. By varying these cones over some natural families, we shall obtain a description of all sets in (1.5) as theta bodies, as well as the corresponding antiblocking duality relations that links them in pairs as in (1.4). We thus unify the description of all these relaxations and show that the ingenious though ad hoc description (1.1) is in fact quite central, powerful, and natural. The corresponding theory shall also make clearer the key role played by the positive semidefinite cone in this generalized context, leading to the most striking of the duality relations (1.4), namely, the extremely symmetric relation (1.4a).

The key axiom we shall need require from our parameter cones $\mathbb{A}$ and $\widehat{\mathbb{K}}$ will be their diagonally scaling invariance, i.e., these cones must be closed under simultaneous left- and right-multiplications by any (and the same) nonnegative diagonal matrix. We shall then generalize the proofs of equivalence of several formulations for $\vartheta$ to rely (essentially) solely on this invariance property. This axiomatic approach also allows us to gauge the full power of the existing proof methods; for instance, some of the equivalent formulations for $\vartheta$ shall only work when the cone $\widehat{\mathbb{K}}$ is the positive semidefinite cone $\mathbb{S}_{+}^{\{0\} \cup V}$.

Another advantage of unifying the equivalent formulations of $\vartheta$ is that it provides less error-prone proofs of equivalent formulations of the variants $\vartheta^{\prime}$ and $\vartheta^{+}$. Many such formulations are listed in the literature 
without proof, with the apparently implicit suggestion that, to prove them, it suffices to repeat and slightly adapt the corresponding proofs for $\vartheta$. While this may be true in most cases, it has already led to some inaccuracies in the literature, as pointed out by the authors in [9, Sec. 4.1].

Finally, we are able to extend the copositive formulation of the fractional chromatic number by Dukanovic and Rendl [14] to the weighted case, and we provide a unified treatment of weighted generalizations of the convex quadratic characterization of $\vartheta$ by Luz and Schrijver [37] to all of $\vartheta, \vartheta^{\prime}$, and $\vartheta^{+}$.

1.1. Organization of the text. The classical monograph [23], which develops much of the theory surrounding the theta function, defines weighted parameters $\vartheta_{i}(G ; w)$ for each $i \in\{1, \ldots, 4\}$ and shows that they are all equal to $\vartheta(G ; w)$ by proving the chain of inequalities

$$
\vartheta(G ; w) \leq \vartheta_{1}(G ; w) \leq \vartheta_{2}(G ; w) \leq \vartheta_{3}(G ; w) \leq \vartheta_{4}(G ; w) \leq \vartheta(G ; w)
$$

for a nonnegative weight function $w$ on $V$; see also [30, Sec. 5]. For instance, $\vartheta_{2}$ is the (weighted generalization of the) formulation on the RHS of (1.3). In this paper, we shall generalize these parameters to arbitrary theta bodies and prove that they are all equal under some mild assumptions. As in [23, this shall establish an antiblocking relation like those in (1.4).

As we briefly hinted just before (1.5), we shall parameterize our theta bodies using two cones, which we usually denote by $\mathbb{A}$ and $\widehat{\mathbb{K}}$; e.g., the membership constraints $X_{i j}=0$ for every $i j \in E$ in (1.1) are replaced with the single membership constraint " $X \in \mathbb{A}$ ". One slightly confusing issue is the fact that the cones $\mathbb{A}$ and $\widehat{\mathbb{K}}$ do not live in the same dimension: $\mathbb{A}$ lives in $\mathbb{S}^{V}$, whereas $\widehat{\mathbb{K}}$ lies in the higher-dimensional cone $\mathbb{S}^{\{0\} \cup V}$, where 0 is assumed not to be in $V$. Throughout the paper, we label subsets of $\mathbb{S}\{0\} \cup V$ with a wide hat, as in $\widehat{\mathbb{K}}$, and elements of such sets with a hat, e.g., $\hat{X} \in \widehat{\mathbb{K}}$.

We shall see later that it is rather natural and convenient to define a "lifting" of a cone $\mathbb{K}$ in $\mathbb{S}^{V}$ to a higher-dimensional cone in $\mathbb{S}\{0\} \cup V$ in a systematic way. In fact, we shall define two such lifting operators for a cone $\mathbb{K} \subseteq \mathbb{S}^{V}$, denoted by $\operatorname{Psd}(\cdot)$ and $\operatorname{Schur}(\cdot)$, in such a way that the antiblocker of a theta body parameterized by $\operatorname{Psd}(\mathbb{K})$ is parameterized by $\operatorname{Schur}(\cdot)$ applied to the dual cone of $\mathbb{K}$. Similarly, we will define a notion of duality for the cone $\mathbb{A}$, that encodes adjacencies, and the "dual" of $\mathbb{A}$ will be denoted by $\overline{\mathbb{A}}$ to match the occurrence of complements of graphs in (1.4).

With these remarks in mind, we may now describe the organization of the paper. We list basic terminology and notation in Subsection 1.2. We then define arbitrary theta bodies and prove their most basic properties in Section 2. In Section 3, we study the structure of the cones $\mathbb{A}$ that make sense in our theory, namely, the ones that are diagonally scaling-invariant and polyhedral; we also quickly develop their duality theory. Next, in Section 4, we describe the lifting operators for cones mentioned above, and we prove a weak duality theorem. Some basic reformulations of antiblocking duality are recalled and adapted to our context in Section 5. The latter three sections also prove a number of equivalences among the $\vartheta_{i}$ 's corresponding to (1.6). These equivalences are put together in Section 6 to prove our generalization of the equivalence (1.6), the duality relations (1.4), and further $\vartheta$ results. In Sections 7 and 9 we study properties of some theta bodies defined over some specific cones, namely, the copositive and completely positive cones, and the semidefinite cone. In between those sections, we describe in Section 8 a further characterization of $\vartheta$ related to Hoffman bounds for the chromatic number of a graph.

1.2. Notation. We set the following notation. Throughout the paper, $V$ shall denote a finite set. We assume throughout that $0 \notin V$, as we will often adjoin 0 to $V$ to form an index set $\{0\} \cup V$. The family of subsets of $V$ of size 2 is denoted by $\left(\begin{array}{c}V \\ 2\end{array}\right)$. If $E \subseteq\left(\begin{array}{c}V \\ 2\end{array}\right)$, we set $\bar{E}:=\left(\begin{array}{c}V \\ 2\end{array}\right) \backslash E$. For distinct $i, j \in V$, we denote $i j:=\{i, j\}$. The standard basis vectors of $\mathbb{R}^{V}$ are $\left\{e_{i}: i \in V\right\}$. We adopt Iverson notation: for a predicate $P$, we denote

$$
[P]:= \begin{cases}1 & \text { if } P \text { holds } \\ 0 & \text { otherwise. }\end{cases}
$$

If $P$ is false, then we consider $[P]$ to be "strongly zero," in the sense that we sometimes write expressions of the form $[x \neq 0](1 / x)$ that evaluate to 0 if $x=0$.

Most of the rest of our notation is listed over tables 10, 2, and 3 ,

We will often consider subsets of $\mathbb{S}^{V}$ and $\mathbb{S}\{0\} \cup V$. Subsets of the latter, as well as their elements, shall be decorated with a hat, e.g., $\hat{X} \in \widehat{\mathbb{K}} \subseteq \mathbb{S}^{\{0\} \cup V}$. If $\mathbb{K} \subseteq \mathbb{E}$ is a pointed closed convex cone with nonempty interior, then $\mathbb{K}$ defines a partial order $\succeq_{\mathbb{K}}$ on $\mathbb{E}$ : we write $x \succeq_{\mathbb{K}} y$ to mean that $x-y \in \mathbb{K}$. We set $\succeq: \succeq_{\mathbb{S}_{+}^{V}}$. 
TABLE 1. Special sets.

\begin{aligned} & \hline $\mathbb{R}_{+}:=\{x \in \mathbb{R}: x \geq 0\} \\ & \mathbb{R}_{++}:=\{x \in \mathbb{R}: x>0\} \\ & \mathbb{S}^{V}:=$ the space of symmetric $V \times V$ matrices \\ & $\mathbb{S}_{+}^{V}:=$ the cone of positive semidefinite matrices in $\mathbb{S}^{V} \\ & \mathbb{S}_{\geq 0}^{V}:=\left\{X \in \mathbb{S}^{V}: X \geq 0\right\}$, the cone of entrywise nonnegative matrices in $\mathbb{S}^{V} \\ & \mathcal{C}_{V}:=\left\{X \in \mathbb{S}^{V}: h^{\top} X h \geq 0 \forall h \in \mathbb{R}_{+}^{V}\right\}$, the cone of copositive matrices \\ & $\mathcal{C}_{V}^{*}:=$ the cone of completely positive matrices, i.e., the dual cone of $\mathcal{C}_{V} \\ &$\hline\end{aligned}

TABLE 2. Notation for vectors and matrices.

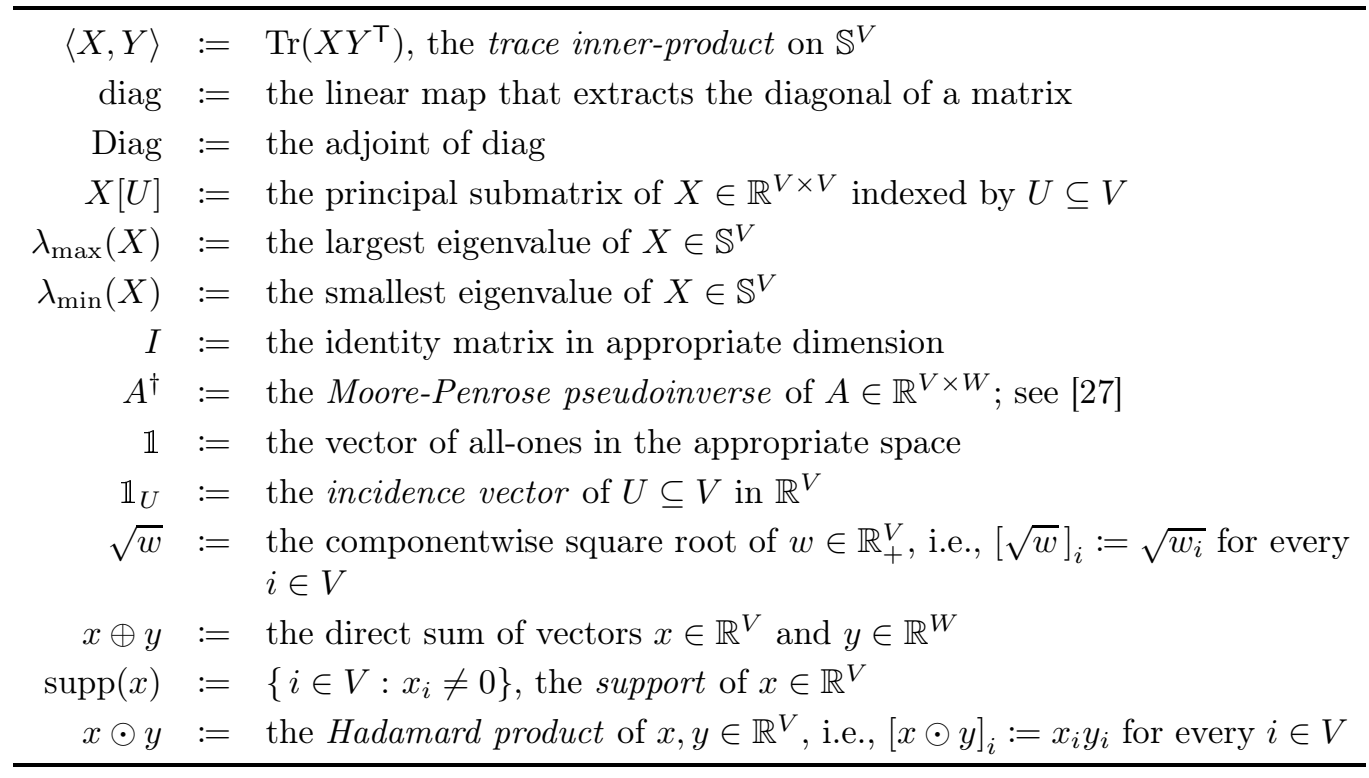

TABle 3. Notation for Convex Analysis, mostly following [41].

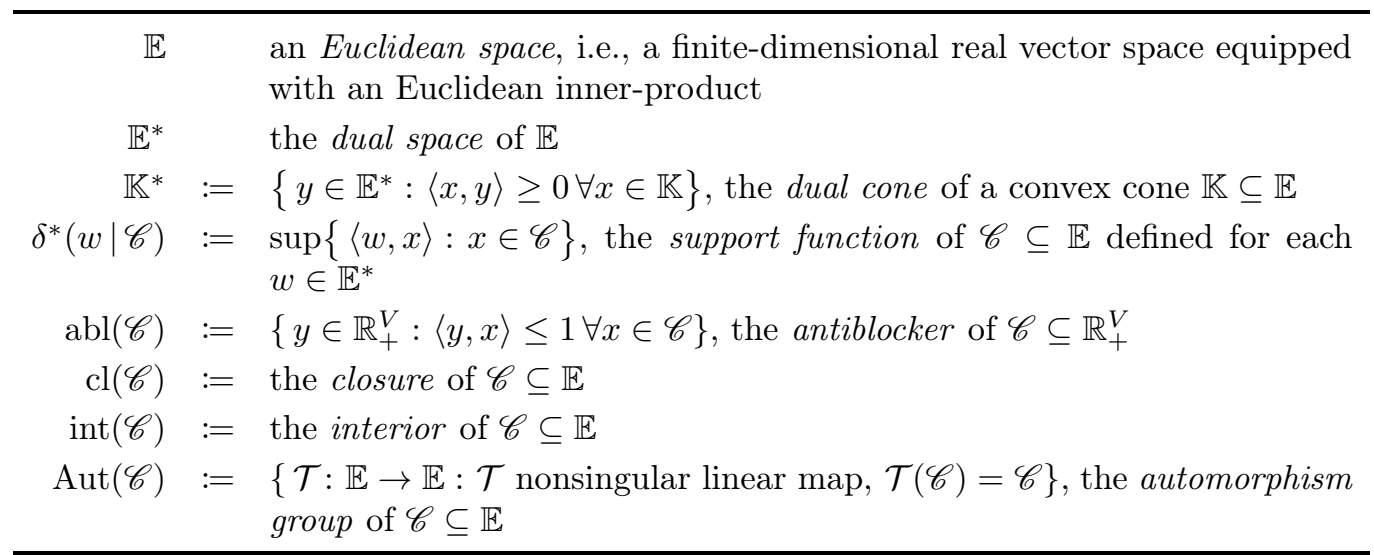

\section{Theta Bodies}

For each $h \in \mathbb{R}^{V}$, define the diagonal scaling map $\mathcal{D}_{h}: \mathbb{R}^{V \times V} \rightarrow \mathbb{R}^{V \times V}$ as

$$
\mathcal{D}_{h}(X):=\operatorname{Diag}(h) X \operatorname{Diag}(h) \quad \forall X \in \mathbb{R}^{V \times V} .
$$


Note that each entry $\left[\mathcal{D}_{h}(X)\right]_{i j}$ is the componentwise product $X_{i j}\left[h h^{\top}\right]_{i j}$ for every $X \in \mathbb{S}^{V}$ and $h \in \mathbb{R}^{V}$. A subset $\mathbb{K}$ of $\mathbb{S}^{V}$ is called diagonally scaling-invariant if $\mathcal{D}_{h}(\mathbb{K}) \subseteq \mathbb{K}$ for every $h \in \mathbb{R}_{+}^{V}$. The cones $\mathbb{S}_{+}^{V}, \mathbb{S}_{\geq 0}^{V}, \mathcal{C}_{V}$, $\mathcal{C}_{V}^{*}$ are all examples of diagonally scaling-invariant subsets of $\mathbb{S}^{V}$. Some other important examples are the sets of the form

$$
\mathbb{A}_{E^{+}, E^{-}}:=\left\{X \in \mathbb{S}^{V}: X_{i j} \geq 0 \forall i j \in E^{+}, X_{i j} \leq 0 \forall i j \in E^{-}\right\},
$$

where $E^{+}, E^{-} \subseteq\left(\begin{array}{c}V \\ 2\end{array}\right)$. Clearly, every diagonally scaling-invariant set is a cone, and since the map $\mathcal{D}_{h}$ is self-adjoint, diagonal scaling invariance is preserved under duality. Moreover, if $\mathbb{K} \subseteq \mathbb{S}^{V}$ is diagonally scaling-invariant, then $\left\{\mathcal{D}_{h}: h \in \mathbb{R}_{++}^{V}\right\} \subseteq \operatorname{Aut}(\mathbb{K})$.

For sets $\mathbb{A} \subseteq \mathbb{S}^{V}$ and $\widehat{\mathbb{K}} \subseteq \mathbb{S}^{\{0\} \cup V}$, define

$$
\widehat{\mathrm{TH}}(\mathbb{A}, \widehat{\mathbb{K}}):=\left\{\hat{X} \in \widehat{\mathbb{K}}: \hat{X}_{00}=1, \hat{X} e_{0}=\operatorname{diag}(\hat{X}), \hat{X}[V] \in \mathbb{A}\right\}
$$

and

$$
\operatorname{TH}(\mathbb{A}, \widehat{\mathbb{K}}):=\{\operatorname{diag}(\hat{X}[V]): \hat{X} \in \widehat{\mathrm{TH}}(\mathbb{A}, \widehat{\mathbb{K}})\} .
$$

We are interested in sets of the form $\mathrm{TH}(\mathbb{A}, \widehat{\mathbb{K}})$, where $\mathbb{A}$ and $\widehat{\mathbb{K}}$ are diagonally scaling-invariant convex cones with a few extra properties. The most important known examples of sets of this form are the theta body $\operatorname{TH}(G)$ of a graph $G=(V, E)$ and its variants $\mathrm{TH}^{\prime}(G)$ and $\mathrm{TH}^{+}(G)$. In fact, we define

$$
\begin{gathered}
\operatorname{TH}(G):=\operatorname{TH}\left(\mathbb{A}_{E, E}, \mathbb{S}_{+}^{\{0\} \cup V}\right), \\
\operatorname{TH}^{\prime}(G):=\operatorname{TH}\left(\mathbb{A}_{E \cup E}, \mathbb{S}_{+}^{\{0\} \cup V}\right), \\
\operatorname{TH}^{+}(G):=\operatorname{TH}\left(\mathbb{A}_{\varnothing, E}, \mathbb{S}_{+}^{\{0\} \cup V}\right) .
\end{gathered}
$$

It thus makes sense to call sets of the form $\mathrm{TH}(\mathbb{A}, \widehat{\mathbb{K}})$ as theta bodies (the terminology "theta bodies" was also used for another generalization of the theta body by [21]; our definition and approach are very different). To avoid confusion, whenever we refer to the specific theta body $\operatorname{TH}(G)$, we shall call it the theta body of $G$.

In the remainder of this section we shall prove that, under certain simple hypotheses, every theta body is a convex corner, i.e., a compact, lower-comprehensive convex subset of the nonnegative orthant with nonempty interior. Recall that a subset $\mathscr{C}$ of $\mathbb{R}_{+}^{V}$ is called lower-comprehensive if, for any $x, y \in \mathbb{R}^{V}$, the chain of relations $0 \leq y \leq x \in \mathscr{C}$ implies $y \in \mathscr{C}$. In what follows, the extra hypotheses (2.6) and (2.7) on $\mathbb{A}$ and $\widehat{\mathbb{K}}$ may be thought of as requiring that $\mathbb{A}$ is not "too small", and that $\widehat{\mathbb{K}}$ is neither "too small" nor "too big."

Proposition 1. Let $\mathbb{A} \subseteq \mathbb{S}^{V}$ and $\widehat{\mathbb{K}} \subseteq \mathbb{S}^{\{0\} \cup V}$ be diagonally scaling-invariant closed convex cones. Suppose that $\mathbb{A}$ satisfies

and suppose that $\widehat{\mathbb{K}}$ satisfies

$$
\operatorname{Im}(\operatorname{Diag}) \subseteq \mathbb{A},
$$

$$
\widehat{\mathbb{K}} \supseteq\left\{\left(e_{0}+e_{i}\right)\left(e_{0}+e_{i}\right)^{\top}: i \in V\right\}
$$

and

$$
\operatorname{diag}\left(\left\{\hat{X} \in \widehat{\mathbb{K}}: \hat{X}_{00}=1, \hat{X} e_{0}=\operatorname{diag}(\hat{X})\right\}\right) \subseteq[0,1]^{\{0\} \cup V} .
$$

Then $\operatorname{TH}(\mathbb{A}, \widehat{\mathbb{K}})$ is a convex lower-comprehensive subset of $[0,1]^{V}$ with nonempty interior. In particular, $\operatorname{cl}(\operatorname{TH}(\mathbb{A}, \widehat{\mathbb{K}}))$ is a convex corner.

Proof. Convexity of the projection $\mathrm{TH}(\mathbb{A}, \widehat{\mathbb{K}})$ follows from that of $\widehat{\mathrm{TH}}(\mathbb{A}, \widehat{\mathbb{K}})$. It is clear from (2.7b) that $\mathrm{TH}(\mathbb{A}, \widehat{\mathbb{K}}) \subseteq[0,1]^{V}$. To prove that the convex set $\mathrm{TH}(\mathbb{A}, \widehat{\mathbb{K}})$ is lower-comprehensive, it suffices to show that if $x \in \mathrm{TH}(\mathbb{A}, \widehat{\mathbb{K}})$ then $x-x_{i} e_{i} \in \mathrm{TH}(\mathbb{A}, \widehat{\mathbb{K}})$ for each $i \in V$. Let $\hat{X} \in \widehat{\mathrm{TH}}(\mathbb{A}, \widehat{\mathbb{K}})$ such that $x=\operatorname{diag}(\hat{X}[V])$. Let $i \in V$. Set $\hat{Y}:=\mathcal{D}_{1 \oplus h}(\hat{X}) \in \widehat{\mathbb{K}}$ for $h:=\mathbb{1}-e_{i} \in \mathbb{R}^{V}$. Then diagonal scaling invariance of $\mathbb{A}$ and $\widehat{\mathbb{K}}$ imply that $\hat{Y} \in \widehat{\mathrm{TH}}(\mathbb{A}, \widehat{\mathbb{K}})$. Thus, $x-x_{i} e_{i}=\operatorname{diag}(\hat{Y}[V]) \in \mathrm{TH}(\mathbb{A}, \widehat{\mathbb{K}})$. This proves that $\mathrm{TH}(\mathbb{A}, \widehat{\mathbb{K}})$ is lowercomprehensive. It remains to show that $\mathrm{TH}(\mathbb{A}, \widehat{\mathbb{K}})$ has nonempty interior. Let $i \in V$. By (2.6), we have $e_{i} e_{i}^{\top} \in \mathbb{A}$. Thus, $\left(e_{0}+e_{i}\right)\left(e_{0}+e_{i}\right)^{\top} \in \widehat{\mathrm{TH}}(\mathbb{A}, \widehat{\mathbb{K}})$ by $(2.7 \mathrm{a})$ whence $e_{i} \in \mathrm{TH}(\mathbb{A}, \widehat{\mathbb{K}})$. Now convexity of $\mathrm{TH}(\mathbb{A}, \widehat{\mathbb{K}})$ implies that $\frac{1}{n} \mathbb{1} \in \mathrm{TH}(\mathbb{A}, \widehat{\mathbb{K}})$, where $n:=|V|$. Since $\mathrm{TH}(\mathbb{A}, \widehat{\mathbb{K}})$ is lower-comprehensive, we find that $\frac{1}{2 n} \mathbb{1} \in \operatorname{int}(\mathrm{TH}(\mathbb{A}, \widehat{\mathbb{K}}))$. 
The reason for using the hypothesis (2.6) rather than the slightly weaker condition $\operatorname{Diag}\left(\mathbb{R}_{+}^{V}\right) \subseteq \mathbb{A}$ shall be made clearer in Section 3, where we develop a notion of duality for such cones. As for the closedness of theta bodies, we are not aware of any example of a theta body that is not closed, so the closure operator in Proposition 1 shall remain as a minor nuisance. We shall now see that, under a mild condition on the cone $\widehat{\mathbb{K}}$, the theta body $\mathrm{TH}(\mathbb{A}, \widehat{\mathbb{K}})$ is actually closed, and hence a convex corner itself.

Corollary 2. Let $\mathbb{A} \subseteq \mathbb{S}^{V}$ and $\widehat{\mathbb{K}} \subseteq \mathbb{S}^{\{0\} \cup V}$ be diagonally scaling-invariant closed convex cones such that (2.6) and (2.7a) hold. If

$$
\widehat{\mathbb{K}} \subseteq\left\{\hat{X} \in \mathbb{S}^{\{0\} \cup V}: \hat{X}[S] \succeq 0, \forall S \in\left(\begin{array}{c}
\{0\} \cup V \\
2
\end{array}\right)\right\},
$$

then $(2.7 \mathrm{~b})$ holds and $\widehat{\mathrm{TH}}(\mathbb{A}, \widehat{\mathbb{K}})$ is compact. In particular, $\mathrm{TH}(\mathbb{A}, \widehat{\mathbb{K}})$ is closed, and hence a convex corner.

Proof. Let $\widehat{\mathbb{M}}$ be the set of all $\hat{X}$ in the RHS of (2.8) such that $\hat{X}_{00}=1$ and $\hat{X} e_{0}=\operatorname{diag}(\hat{X})$. Then $\widehat{\mathbb{M}}$ is bounded. To see this, first use sets $S \in\left(\begin{array}{c}\{0\} \cup V \\ 2\end{array}\right)$ containing 0 to show that $\operatorname{diag}(\widehat{\mathbb{M}}) \subseteq[0,1]^{\{0\} \cup V}$. Thus, (2.7b) holds. Next, use (2.8) with sets $S \in\left(\begin{array}{c}V \\ 2\end{array}\right)$ to show that all off-diagonal entries of $\hat{X} \in \widehat{\mathbb{M}}$ have absolute value bounded above by 1 . Since $\widehat{\mathrm{TH}}(\mathbb{A}, \widehat{\mathbb{K}}) \subseteq \widehat{\mathbb{M}}$, it follows that $\widehat{\mathrm{TH}}(\mathbb{A}, \widehat{\mathbb{K}})$ is compact. Now closedness of $\mathrm{TH}(\mathbb{A}, \widehat{\mathbb{K}})$ follows from the fact that $\mathrm{TH}(\mathbb{A}, \widehat{\mathbb{K}})$ is a linear image of the compact set $\widehat{\mathrm{TH}}(\mathbb{A}, \widehat{\mathbb{K}})$. The rest follows from Proposition 1 .

For most of the theta bodies in this paper, the cone $\widehat{\mathbb{K}}$ shall be a subset of $\mathbb{S}_{+}^{\{0\} \cup V}$, and hence (2.8) shall be satisfied; this already includes the theta bodies described in (2.5). An important diagonally scalinginvariant closed convex cone which does not satisfy (2.8) is the cone of copositive matrices; indeed, note that $\mathcal{C}_{\{0\} \cup V} \supseteq \mathbb{S}_{\geq 0}^{\{0\} \cup V}$. We shall deal with theta bodies arising from the copositive cone in Section 7 , where we shall prove directly that the corresponding theta body is closed.

\section{Polyhedral Diagonally Scaling-Invariant Cones}

When studying a theta body $\mathrm{TH}(\mathbb{A}, \widehat{\mathbb{K}})$, we think of $\mathbb{A}$ as an "elementary" cone, while $\widehat{\mathbb{K}}$ is (potentially) a "sophisticated" cone. In the most important instances of theta bodies, namely the ones described in (2.5), the cone $\mathbb{A}$ is polyhedral, whereas $\widehat{\mathbb{K}}$ is the nonlinear cone $\mathbb{S}_{+}^{\{0\} \cup V}$. In general, it makes sense to focus on the case where $\mathbb{A}$ is polyhedral. At any rate, when defining a theta body $\mathrm{TH}(\mathbb{A}, \widehat{\mathbb{K}})$, any trace of "non-polyhedrality" may be (and should be) "pushed" away from $\mathbb{A}$ and into $\widehat{\mathbb{K}}$. We shall show next that requiring a closed convex cone to be both diagonally scaling-invariant and polyhedral severely constrains its structure.

We shall need a family of cones slightly more refined than the cones $\mathbb{A}_{E^{+}, E^{-}}$defined in (2.2). Let $V^{+}, V^{-} \subseteq V$ and $E^{+}, E^{-} \subseteq\left(\begin{array}{c}V \\ 2\end{array}\right)$. Define

$$
\mathbb{A}_{V^{+}, V^{-}, E^{+}, E^{-}}:=\left\{X \in \mathbb{A}_{E^{+}, E^{-}}: \operatorname{diag}\left(X\left[V^{+}\right]\right) \geq 0, \operatorname{diag}\left(X\left[V^{-}\right]\right) \leq 0\right\} .
$$

Clearly, every set of this form is diagonally scaling-invariant and polyhedral. In fact, every polyhedral diagonally scaling-invariant cone is of this form:

Proposition 3. Let $\mathbb{A} \subseteq \mathbb{S}^{V}$ be a diagonally scaling-invariant closed convex cone. If $\mathbb{A}$ is polyhedral, then $\mathbb{A}$ is of the form $\mathbb{A}=\mathbb{A}_{V^{+}, V^{-}, E^{+}, E^{-}}$for some subsets $V^{+}, V^{-} \subseteq V$ and $E^{+}, E^{-} \subseteq\left(\begin{array}{c}V \\ 2\end{array}\right)$.

Proof. Let Sym: $\mathbb{R}^{V \times V} \rightarrow \mathbb{S}^{V}$ denote the orthogonal projection onto $\mathbb{S}^{V}$, that is,

$$
\operatorname{Sym}(X):=\frac{1}{2}\left(X+X^{\top}\right) \quad \forall X \in \mathbb{R}^{V \times V} .
$$

It suffices to show that

$$
\text { every extreme ray of } \mathbb{A}^{*} \text { is of the form } \pm \mathbb{R}_{+} \operatorname{Sym}\left(e_{i} e_{j}^{\top}\right) \text { for some } i, j \in V \text {. }
$$

We first show that,

$$
\text { if } \mathbb{R}_{+} X \text { is an extreme ray of } \mathbb{A}^{*} \text {, then }|\operatorname{supp}(\operatorname{diag}(X))| \leq 1 \text {. }
$$

Suppose that $\mathbb{R}_{+} X$ is an extreme ray of $\mathbb{A}^{*}$ such that $X_{i i} \neq 0 \neq X_{j j}$ for distinct $i, j \in V$. Since $\mathbb{A}^{*}$ is also diagonally scaling-invariant, we have $\left\{\mathcal{D}_{h}: h \in \mathbb{R}_{++}^{V}\right\} \subseteq \operatorname{Aut}\left(\mathbb{A}^{*}\right)$. For $t \in \mathbb{R}_{++}$, define $h(t):=$ $t e_{i}+t^{-1} e_{j}+\mathbb{1}_{V \backslash\{i, j\}}$. Thus, $\left\{\mathbb{R}_{+} \mathcal{D}_{h(t)}(X): t \in \mathbb{R}_{++}\right\}$is an infinite set of extreme rays of $\mathbb{A}^{*}$. This contradicts the fact that $\mathbb{A}^{*}$ is polyhedral and thus proves (3.3). 
To prove (3.2), let $\mathbb{R}_{+} X$ be an extreme ray of $\mathbb{A}^{*}$. Let us show that

$$
X_{i j} \operatorname{Sym}\left(e_{i} e_{j}^{\top}\right) \in \mathbb{A}^{*} \quad \forall i, j \in V .
$$

Let $i, j \in V$. If $i=j$ then (3.4) holds by diagonal scaling invariance of $\mathbb{A}$, so assume $i \neq j$. By (3.3), at most one of $X_{i i}$ and $X_{j j}$ is nonzero. We may assume by symmetry that $X_{i i}=0$. For $t \in \mathbb{R}_{++}$, define $h(t):=t e_{i}+t^{-1} e_{j}$ and note that $\mathcal{D}_{h(t)}(X) \in \mathbb{A}^{*}$ for every $t \in \mathbb{R}_{++}$. By driving $t$ to $\infty$ we find that $2 X_{i j} \operatorname{Sym}\left(e_{i} e_{j}^{\top}\right)=\lim _{t \rightarrow \infty} \mathcal{D}_{h(t)}(X)$ lies in the closed set $\mathbb{A}^{*}$. This proves (3.4).

Since

$$
X=\sum_{i, j \in V} 2^{[i \neq j]} X_{i j} \operatorname{Sym}\left(e_{i} e_{j}^{\top}\right)
$$

and $\mathbb{R}_{+} X$ is an extreme ray of $\mathbb{A}^{*}$, it follows from (3.4) that at most one of the terms in the RHS of (3.5) is nonzero. This proves (3.2) and concludes the proof.

It follows that a polyhedral diagonally scaling-invariant cone $\mathbb{A} \subseteq \mathbb{S}^{V}$ such that $\operatorname{Im}(\operatorname{Diag}) \subseteq \mathbb{A}$ must have the form shown in (2.2). We shall focus our attention on such cones for the first argument of $\widehat{\mathrm{TH}}$ for the remainder of the paper.

We shall define some alternative notions of "duality" for the cones $\mathbb{A}$ and $\widehat{\mathbb{K}}$ to describe more conveniently the antiblockers of theta bodies. The appropriate duality notion for the cones $\mathbb{A} \subseteq \mathbb{S}^{V}$ is defined as follows:

$$
\overline{\mathbb{A}}:=\operatorname{Im}(\operatorname{Diag})-\mathbb{A}^{*} .
$$

It is easy to check that, for every $E^{+}, E^{-} \subseteq\left(\begin{array}{c}V \\ 2\end{array}\right)$, we have

$$
\overline{\mathbb{A}_{E^{+}, E^{-}}}=\mathbb{A}_{\overline{E^{+}}, \overline{E^{-}}}
$$

and, for every polyhedral diagonally scaling-invariant cone $\mathbb{A} \subseteq \mathbb{S}^{V}$, we have $\overline{\overline{\mathbb{A}}}=\mathbb{A}+\operatorname{Im}($ Diag), so this operation is an involution when restricted to cones that contain $\operatorname{Im}(\mathrm{Diag})$.

\section{Liftings of CONES}

In this section, we define two operators that lift a cone in $\mathbb{S}^{V}$ to a cone in $\mathbb{S}\{0\} \cup V$, as we briefly mentioned in Subsection 1.1. Note that, in the definition (2.3), whenever we test membership of a matrix $\hat{X}$ in $\widehat{\mathbb{K}}$, the 0 th column of $\hat{X}$ is completely determined by $\hat{X}[V]$. Thus, it makes some sense to define a lifting of a cone $\mathbb{K}$ in $\mathbb{S}^{V}$ as a cone in $\mathbb{S}\{0\} \cup V$ in such a way that the 0 th column is strongly related to $\mathbb{K}$. We shall need two such liftings, which are studied in the next two subsections. We shall also see a glimpse of the duality relation involving these two liftings in a Weak Duality theorem, as well as a natural description of the set $\operatorname{FRAC}(G)$ (that appeared in (1.5)) as a theta body using one of the liftings.

4.1. PSD Liftings of Cones. In this subsection, we define the PSD lifting of a cone $\mathbb{K}$ in $\mathbb{S}^{V}$ and we prove that, under mild hypotheses, the support function of a theta body defined over the PSD lifting of $\mathbb{K}$ can be formulated as a simple conic optimization problem over $\mathbb{K}$. This shall correspond to the equivalence $\vartheta_{3}=\vartheta_{4}$ in (1.6).

Let $\mathbb{K} \subseteq \mathbb{S}^{V}$. Define the PSD lifting of $\mathbb{K}$ as

$$
\operatorname{Psd}(\mathbb{K}):=\left\{\hat{X} \in \mathbb{S}_{+}^{\{0\} \cup V}: \hat{X}[V] \in \mathbb{K}\right\} .
$$

Note that if $\mathbb{K}$ is diagonally scaling-invariant, then so is $\operatorname{Psd}(\mathbb{K})$. Moreover, $\operatorname{Psd}\left(\mathbb{S}_{+}^{V}\right)=\mathbb{S}_{+}^{\{0\} \cup V}$.

Before using PSD liftings, we shall need the following straightforward weighted generalization of [19, Proposition 9].

Lemma 4. Let $\mathbb{M} \subseteq \mathbb{S}^{V}$ be a diagonally scaling-invariant closed convex cone. Suppose that

$$
\begin{gathered}
\operatorname{diag}(\mathbb{M}) \subseteq \mathbb{R}_{+}^{V}, \\
\text { if } X_{i i}=0 \text { for some } X \in \mathbb{M} \text { and } i \in V, \text { then } X e_{i}=0, \\
\{X \in \mathbb{M}: \operatorname{Tr}(X)=1\} \text { is compact. }
\end{gathered}
$$

Let $w \in \mathbb{R}_{+}^{V}$. Let $X^{*}$ be an optimal solution of

$$
\max \left\{\sqrt{w}^{\top} X \sqrt{w}: \operatorname{Tr}(X)=1, X \in \mathbb{M}\right\},
$$


and suppose that $\sqrt{w}^{\top} X^{*} \sqrt{w}>0$. Set

$$
\begin{gathered}
d:=\operatorname{diag}\left(X^{*}\right), \\
\bar{X}:=\operatorname{Diag}(\sqrt{d})^{\dagger} X^{*} \operatorname{Diag}(\sqrt{d})^{\dagger}, \\
\lambda:=\lambda_{\max }\left(\mathcal{D}_{\sqrt{w}}(\bar{X})\right) .
\end{gathered}
$$

Then

$$
\begin{gathered}
\operatorname{supp}(d) \subseteq \operatorname{supp}(w), \\
\mathcal{D}_{\sqrt{w}}(\bar{X}) \sqrt{d}=\lambda \sqrt{d}, \\
\lambda=\sqrt{w}^{\top} X^{*} \sqrt{w}, \\
X^{*} \sqrt{w}=\lambda \operatorname{Diag}(\sqrt{w})^{\dagger} d .
\end{gathered}
$$

Proof. We first show (4.4a). Let $i \in \operatorname{supp}(d)$, so that $X_{i i}^{*}>0$. Suppose that $w_{i}=0$. If $X_{i i}^{*}=1$, then $X^{*}=e_{i} e_{i}^{\top}$ by (4.2) whence $\sqrt{w}^{\top} X^{*} \sqrt{w}=0$. If $X_{i i}^{*}<1$, then $\left(1-X_{i i}^{*}\right)^{-1} \mathcal{D}_{\mathbb{1}-e_{i}}\left(X^{*}\right)$ is feasible for (4.3) with objective value $\left(1-X_{i i}^{*}\right)^{-1} \sqrt{w}^{\top} X^{*} \sqrt{w}$, hence strictly larger than the objective value of $X^{*}$. In either case, we get a contradiction. This proves (4.4a).

If $d_{i}=0$ for some $i \in V$, we are done by induction on $|V|$. Thus, from (4.4a) we may assume that

$$
\operatorname{supp}(d)=\operatorname{supp}(w)=V \text {. }
$$

Define $d^{-1 / 2} \in \mathbb{R}^{V}$ such that $d^{-1 / 2} \odot \sqrt{d}=\mathbb{1}$, so $\bar{X}=\mathcal{D}_{d^{-1 / 2}}\left(X^{*}\right) \in \mathbb{M}$ and $\operatorname{diag}(\bar{X})=\mathbb{1}$. For every $h \in \mathbb{R}_{+}^{V}$ with $\|h\|=1$, the point $\mathcal{D}_{h}(\bar{X})$ is feasible for (4.3) with objective value $\sqrt{w}^{\top} \mathcal{D}_{h}(\bar{X}) \sqrt{w}=$ $h^{\top} \mathcal{D}_{\sqrt{w}}(\bar{X}) h$. Since $X^{*}=\mathcal{D}_{\sqrt{d}}(\bar{X})$ is optimal for (4.3), it follows that $\sqrt{d}$ is an optimal solution for $\max \left\{h^{\top} \mathcal{D}_{\sqrt{w}}(\bar{X}) h: h \in \mathbb{R}_{+}^{V},\|h\|=1\right\}$. In fact, since $[\sqrt{d}]_{i}>0$ for all $i \in V$, we find that $\sqrt{d}$ is a local optimal solution for $\max \left\{h^{\top} \mathcal{D}_{\sqrt{w}}(\bar{X}) h: h \in \mathbb{R}^{V},\|h\|=1\right\}$, hence also a global one (note that the sign of $h$ is unconstrained here). Thus, $\mathcal{D}_{\sqrt{w}}(\bar{X}) \sqrt{d}=\lambda \sqrt{d}$. This proves (4.4b). Now we unroll:

$$
\begin{aligned}
\lambda d & =\lambda \operatorname{Diag}(\sqrt{d}) \sqrt{d}=\operatorname{Diag}(\sqrt{d}) \mathcal{D}_{\sqrt{w}}\left(\mathcal{D}_{d^{-1 / 2}}\left(X^{*}\right)\right) \sqrt{d}=\operatorname{Diag}(\sqrt{d}) \mathcal{D}_{d^{-1 / 2}}\left(\mathcal{D}_{\sqrt{w}}\left(X^{*}\right)\right) \sqrt{d} \\
& =\operatorname{Diag}(\sqrt{d}) \operatorname{Diag}\left(d^{-1 / 2}\right) \mathcal{D}_{\sqrt{w}}\left(X^{*}\right) \operatorname{Diag}\left(d^{-1 / 2}\right) \sqrt{d}=\operatorname{Diag}(\sqrt{w}) X^{*} \operatorname{Diag}(\sqrt{w}) \mathbb{1}=\operatorname{Diag}(\sqrt{w}) X^{*} \sqrt{w} .
\end{aligned}
$$

This proves (4.4d). Finally, $\lambda=\lambda \operatorname{Tr}\left(X^{*}\right)=\lambda \mathbb{1}^{\top} d=\mathbb{1}^{\top} \operatorname{Diag}(\sqrt{w}) X^{*} \sqrt{w}=\sqrt{w}^{\top} X^{*} \sqrt{w}$ so (4.4C) is proved.

We can now show that the support function of some theta bodies of the form $\operatorname{TH}(\mathbb{A}, \operatorname{Psd}(\mathbb{K}))$, which shall correspond to $\vartheta_{4}$ in (1.6), may be formulated as a conic optimization problem over $\mathbb{K}$; the latter shall correspond to $\vartheta_{3}$ from (1.6). Note that the next result does not make use of Duality Theory.

Theorem 5. Let $\mathbb{A} \subseteq \mathbb{S}^{V}$ and $\mathbb{K} \subseteq \mathbb{S}_{+}^{V}$ be diagonally scaling-invariant closed convex cones such that $\operatorname{Im}(\operatorname{Diag}) \subseteq \mathbb{A}$ and $\operatorname{Diag}\left(\mathbb{R}_{+}^{V}\right) \subseteq \mathbb{K}$. Let $w \in \mathbb{R}_{+}^{V}$. Then

$$
\delta^{*}(w \mid \operatorname{TH}(\mathbb{A}, \operatorname{Psd}(\mathbb{K})))=\max \left\{\left\langle\sqrt{w} \sqrt{w}^{\top}, X\right\rangle:\langle I, X\rangle=1, X \in \mathbb{A}, X \in \mathbb{K}\right\} .
$$

Moreover, both optimization problems in (4.6) have optimal solutions.

Proof. We begin by proving ' $\leq$ '. Let $y \in \mathrm{TH}(\mathbb{A}, \operatorname{Psd}(\mathbb{K}))$ and let $\hat{Y} \in \widehat{\mathrm{TH}}(\mathbb{A}, \operatorname{Psd}(\mathbb{K}))$ such that $y=\operatorname{diag}(Y)$ for $Y:=\hat{Y}[V]$. We will show that there exists a feasible solution $X$ for the RHS of (4.6) with objective value at least $\langle w, y\rangle$. We may assume that $\langle w, y\rangle>0$; otherwise, take $X=e_{i} e_{i}^{\top}$ for any $i \in V$. Set $h:=\langle w, y\rangle^{-1 / 2} \sqrt{w} \geq 0$ and $X:=\mathcal{D}_{h}(Y) \in \mathbb{A} \cap \mathbb{K}$. Then $\operatorname{Tr}(X)=\langle w, y\rangle^{-1} \operatorname{Tr}\left(\mathcal{D}_{\sqrt{w}}(Y)\right)=\langle w, y\rangle^{-1}\langle\sqrt{w} \odot$ $\sqrt{w}, \operatorname{diag}(Y)\rangle=1$, whence $X$ is feasible on the RHS of (4.6). Moreover,

$$
\left[\begin{array}{cc}
1 & (h \odot y)^{\top} \\
h \odot y & X
\end{array}\right]=\mathcal{D}_{1 \oplus h}\left(\left[\begin{array}{cc}
1 & y^{\top} \\
y & Y
\end{array}\right]\right) \in \mathcal{D}_{1 \oplus h}(\operatorname{Psd}(\mathbb{K})) \subseteq \operatorname{Psd}(\mathbb{K}) \subseteq \mathbb{S}_{+}^{\{0\} \cup V} .
$$

Thus, by Schur complement, we get $X \succeq\langle w, y\rangle^{-1}(\sqrt{w} \odot y)(\sqrt{w} \odot y)^{\top}$ and so

$$
\sqrt{w}^{\top} X \sqrt{w} \geq\langle w, y\rangle^{-1} \sqrt{w}^{\top}(\operatorname{Diag}(\sqrt{w}) y)(\operatorname{Diag}(\sqrt{w}) y)^{\top} \sqrt{w}=\frac{1}{\langle w, y\rangle}\langle w, y\rangle^{2} .
$$


This completes the proof of ' $\leq$ '.

Now we prove ' $\geq$ '. For that, we will show that,

if $X \in \mathbb{A} \cap \mathbb{K}$ and $X \sqrt{w} \geq 0$, then $\sqrt{w}^{\top} X \sqrt{w} \leq[\operatorname{Tr}(X)]\langle w, y\rangle$ for some $y \in \operatorname{TH}(\mathbb{A}, \operatorname{Psd}(\mathbb{K}))$.

So, let $X \in \mathbb{A} \cap \mathbb{K}$ such that $X \sqrt{w} \geq 0$. We may assume that $\sqrt{w}^{\top} X \sqrt{w}>0$; otherwise take $y=0$. Since $X \in \mathbb{K} \subseteq \mathbb{S}_{+}^{V}$, there exists $B \in \mathbb{R}^{V \times V}$ such that $X=B^{\top} B$. Define

$$
\begin{gathered}
c:=\left(\sqrt{w}^{\top} X \sqrt{w}\right)^{-1 / 2} B \sqrt{w}, \\
d:=\operatorname{diag}(X), \\
\tilde{B}:=B[\operatorname{Diag}(\sqrt{d})]^{\dagger}, \\
\bar{B}:=\tilde{B} \operatorname{Diag}\left(\tilde{B}^{\top} c\right), \\
y:=\bar{B}^{\top} c=\operatorname{Diag}\left(\tilde{B}^{\top} c\right) \tilde{B}^{\top} c=\left(\tilde{B}^{\top} c\right) \odot\left(\tilde{B}^{\top} c\right) .
\end{gathered}
$$

We will show that

$$
y \in \operatorname{TH}(\mathbb{A}, \operatorname{Psd}(\mathbb{K})) .
$$

Set $Y:=\bar{B}^{\top} \bar{B}$ and note that

$$
\hat{Y}:=\left[\begin{array}{cc}
1 & y^{\top} \\
y & Y
\end{array}\right]=\left[\begin{array}{cc}
1 & c^{\top} \bar{B} \\
\bar{B}^{\top} c & \bar{B}^{\top} \bar{B}
\end{array}\right]=\left[\begin{array}{c}
c^{\top} \\
\bar{B}^{\top}
\end{array}\right]\left[\begin{array}{cc}
c & \bar{B}
\end{array}\right] \in \operatorname{Psd}(\mathbb{K})
$$

to see that $Y$ lies in $\mathbb{K} \cap \mathbb{A}$, note that $Y=\mathcal{D}_{h}(X)$ for some $h \geq 0$ since

$$
\tilde{B}^{\top} c \geq 0,
$$

which follows from $\left(\sqrt{w}^{\top} X \sqrt{w}\right)^{1 / 2} \tilde{B}^{\top} c=[\operatorname{Diag}(\sqrt{d})]^{\dagger} B^{\top} B \sqrt{w}=[\operatorname{Diag}(\sqrt{d})]^{\dagger} X \sqrt{w} \geq 0$. Finally,

$$
\begin{aligned}
\operatorname{diag}(Y) & =\operatorname{diag}\left(\bar{B}^{\top} \bar{B}\right)=\operatorname{diag}\left(\operatorname{Diag}\left(\tilde{B}^{\top} c\right) \tilde{B}^{\top} \tilde{B} \operatorname{Diag}\left(\tilde{B}^{\top} c\right)\right) \\
& =\left(\tilde{B}^{\top} c\right) \odot \operatorname{diag}\left(\tilde{B}^{\top} \tilde{B}\right) \odot\left(\tilde{B}^{\top} c\right) \\
& =\left(\tilde{B}^{\top} c\right) \odot \operatorname{diag}\left([\operatorname{Diag}(\sqrt{d})]^{\dagger} B^{\top} B[\operatorname{Diag}(\sqrt{d})]^{\dagger}\right) \odot\left(\tilde{B}^{\top} c\right) \\
& =\left(\tilde{B}^{\top} c\right) \odot \mathbb{1}_{\operatorname{supp}(d)} \odot\left(\tilde{B}^{\top} c\right)=\left(\tilde{B}^{\top} c\right) \odot\left(\tilde{B}^{\top} c\right)=y
\end{aligned}
$$

where we used for the second-to-last equation the fact that $d_{j}=0$ implies that $\left(\tilde{B}^{\top} c\right)_{j}=e_{j}^{\top} \tilde{B}^{\top} c=$ $e_{j}^{\top}[\operatorname{Diag}(\sqrt{d})]^{\dagger} B^{\top} c=0^{\top} B^{\top} c=0$. Thus, $\hat{Y} \in \widehat{T H}(\mathbb{A}, \operatorname{Psd}(\mathbb{K}))$, and (4.8) is proved.

We also have $\tilde{B} \operatorname{Diag}(\sqrt{d})=B[\operatorname{Diag}(\sqrt{d})]^{\dagger} \operatorname{Diag}(\sqrt{d})=B \operatorname{Diag}\left(\mathbb{1}_{\operatorname{supp}(d)}\right)=B$ since $d_{i}=0$ implies $B e_{i}=0$. Thus,

$$
\begin{aligned}
\sqrt{w}^{\top} X \sqrt{w} & =\left(\frac{\sqrt{w}^{\top} B^{\top} B \sqrt{w}}{\left(\sqrt{w}^{\top} X \sqrt{w}\right)^{1 / 2}}\right)^{2}=\left(\sqrt{w}^{\top} B^{\top} c\right)^{2} \\
& =\left(\sqrt{w}^{\top} \operatorname{Diag}(\sqrt{d}) \tilde{B}^{\top} c\right)^{2}=\left(\sqrt{d}^{\top} \operatorname{Diag}(\sqrt{w}) \sqrt{y}\right)^{2} \\
& \leq\|\sqrt{d}\|^{2}\|\operatorname{Diag}(\sqrt{w}) \sqrt{y}\|^{2}=[\operatorname{Tr}(X)]\langle w, y\rangle .
\end{aligned}
$$

This completes the proof of (4.7).

Let $X$ be an optimal solution for the RHS of (4.6); the latter set is compact since it is a closed subset of $\left\{X \in \mathbb{S}_{+}^{V}: \operatorname{Tr}(X)=1\right\}$. By Lemma 4, we have $X \sqrt{w} \geq 0$. Thus, $\delta^{*}(w \mid \operatorname{TH}(\mathbb{A}, \operatorname{Psd}(\mathbb{K}))) \geq \sqrt{w}^{\top} X \sqrt{w}$ by (4.7) and the proof of ' $\geq$ ' is complete.

The latter paragraph showed that the RHS is attained. Attainment of the LHS also follows by compactness by Corollary 2 
4.2. Schur Liftings of Cones. In this subsection, we define a lifting operator that is, in a sense, dual to the PSD lifting introduced in the previous subsection. After proving that the Schur lifting of certain convex cones are also convex, we shall prove a Weak Duality result relating both liftings, and then we shall describe $\operatorname{FRAC}(G)$ as a theta body over the Schur lifting of a simple cone.

Let $\mathbb{K} \subseteq \mathbb{S}^{V}$. Define the Schur lifting of $\mathbb{K}$ as

$$
\operatorname{Schur}(\mathbb{K}):=\left\{\left[\begin{array}{cc}
x_{0} & x^{\top} \\
x & X
\end{array}\right] \in \mathbb{S}^{\{0\} \cup V}: X \in \mathbb{K}, x_{0} \in \mathbb{R}_{+}, x_{0} X \succeq_{\mathbb{K}} x x^{\top}\right\} .
$$

Note that $\operatorname{Schur}\left(\mathbb{S}_{+}^{V}\right)=\mathbb{S}_{+}^{\{0\} \cup V}$. It is instructive to rewrite the PSD lifting Psd( $\left.\mathbb{K}\right)$ in the following format similar to $\operatorname{Schur}(\mathbb{K})$ :

$$
\text { if } \mathbb{K} \subseteq \mathbb{S}_{+}^{V} \text {, then } \operatorname{Psd}(\mathbb{K})=\left\{\left[\begin{array}{cc}
x_{0} & x^{\top} \\
x & X
\end{array}\right] \in \mathbb{S}^{\{0\} \cup V}: X \in \mathbb{K}, x_{0} \in \mathbb{R}_{+}, x_{0} X \succeq x x^{\top}\right\} ;
$$

note the difference in the last (conic) inequality.

Whereas the expression (4.1) makes it clear that the PSD lifting of a closed convex cone is convex, the same can not be said about the Schur lifting. We shall now show that, under certain simple conditions, the Schur lifting of a convex cone is also convex, and in fact it satisfies the properties (2.7) of the cone $\widehat{\mathbb{K}}$ in Proposition [1]

Theorem 6. Let $\mathbb{K} \subseteq \mathbb{S}^{V}$ be a diagonally scaling-invariant closed convex cone such that $\mathbb{K} \supseteq \mathbb{S}_{+}^{V}$ and $\operatorname{diag}(\mathbb{K}) \subseteq \mathbb{R}_{+}^{V}$. Then $\operatorname{Schur}(\mathbb{K})$ is a diagonally scaling-invariant closed convex cone that satisfies (2.7). In particular, if $\mathbb{A} \subseteq \mathbb{S}^{V}$ is a diagonally scaling-invariant closed convex cone such that (2.6) holds, then $\operatorname{cl}(\operatorname{TH}(\mathbb{A}, \operatorname{Schur}(\mathbb{K})))$ is a convex corner contained in $[0,1]^{V}$.

Proof. Closedness of $\operatorname{Schur}(\mathbb{K})$ follows from that of $\mathbb{K}$. Using $\operatorname{diag}(\mathbb{K}) \subseteq \mathbb{R}_{+}^{V}$, it is not hard to check that

$$
\operatorname{Schur}(\mathbb{K})=\operatorname{cl}(\widehat{\mathbb{M}}),
$$

where

$$
\widehat{\mathbb{M}}:=\left\{\left[\begin{array}{cc}
x_{0} & x^{\top} \\
x & X
\end{array}\right] \in \mathbb{S}^{\{0\} \cup V}: X \in \mathbb{K}, x_{0} \in \mathbb{R}_{++}, x_{0} X \succeq_{\mathbb{K}} x x^{\top}\right\} .
$$

It is obvious that $\operatorname{Schur}(\mathbb{K})$ is a cone. We shall prove that $\operatorname{Schur}(\mathbb{K})$ is convex by showing that

$$
\widehat{\mathbb{M}} \text { is convex. }
$$

Since

$$
\widehat{\mathbb{M}}=\left\{\left[\begin{array}{cc}
x_{0} & x^{\top} \\
x & X
\end{array}\right] \in \mathbb{S}^{\{0\} \cup V}: X \in \mathbb{K}, x_{0} \in \mathbb{R}_{++},\left\langle H, x_{0} X-x x^{\top}\right\rangle \geq 0 \forall H \in \mathbb{K}^{*}\right\},
$$

it suffices to show that, for each $H \in \mathbb{K}^{*}$,

$$
\text { the function } f_{H}: x_{0} \oplus x \in \mathbb{R}_{++} \oplus \mathbb{R}^{V} \mapsto \frac{x^{\top} H x}{x_{0}} \text { is convex. }
$$

Let $H \in \mathbb{K}^{*}$. The Hessian of $f_{H}$ is

$$
\nabla^{2} f_{H}\left(x_{0} \oplus x\right)=\frac{2}{x_{0}^{2}}\left[\begin{array}{cc}
x^{\top} H x / x_{0} & -(H x)^{\top} \\
-H x & x_{0} H
\end{array}\right] .
$$

From the hypothesis that $\mathbb{K} \supseteq \mathbb{S}_{+}^{V}$ we get $\mathbb{K}^{*} \subseteq \mathbb{S}_{+}^{V}$ whence $H \succeq 0$, so we may write $H=\sum_{h \in \mathscr{H}} h h^{\top}$ for a finite subset $\mathscr{H}$ of $\mathbb{R}^{V}$. For $u:=x_{0}^{1 / 2} \oplus x_{0}^{-1 / 2} \mathbb{1} \in \mathbb{R}_{++}^{\{0\}} \oplus \mathbb{R}_{++}^{V}$, we have

$$
\frac{x_{0}^{2}}{2} \mathcal{D}_{u}\left(\nabla^{2} f_{H}\left(x_{0} \oplus x\right)\right)=\sum_{h \in \mathscr{H}} \mathcal{D}_{\langle h, x\rangle \oplus \mathbb{1}}\left(\left[\begin{array}{cc}
1 & -h^{\top} \\
-h & h h^{\top}
\end{array}\right]\right) \succeq 0 .
$$

Thus, $\nabla^{2} f_{H}\left(x_{0} \oplus x\right) \succeq 0$, and this concludes the proofs of (4.15) and (4.14). Therefore, Schur( $\mathbb{K}$ ) is convex by (4.13).

Let

$$
\hat{X}:=\left[\begin{array}{cc}
x_{0} & x^{\top} \\
x & X
\end{array}\right] \in \operatorname{Schur}(\mathbb{K}),
$$

and let $h_{0} \oplus h \in \mathbb{R}_{+}^{\{0\}} \oplus \mathbb{R}_{+}^{V}$. The condition $\mathcal{D}_{h_{0} \oplus h}(\hat{X}) \in \operatorname{Schur}(\mathbb{K})$ is equivalent to $\mathcal{D}_{h}(X) \in \mathbb{K}$ and $h_{0}^{2} x_{0} \mathcal{D}_{h}(X) \succeq_{\mathbb{K}} h_{0}^{2} \mathcal{D}_{h}\left(x x^{\boldsymbol{\top}}\right)$, both of which follow from the diagonal scaling invariance of $\mathbb{K}$. It is easy to 
check that $\operatorname{Schur}(\mathbb{K})$ satisfies (2.7a). For (2.7b), note that $x-(x \odot x)=\operatorname{diag}\left(X-x x^{\top}\right) \geq 0$ since $\operatorname{diag}(\mathbb{K}) \subseteq \mathbb{R}_{+}^{V}$ whence $x \subseteq[0,1]^{V}$. This completes the proof that (2.7) holds. The remainder of the statement of the theorem follows from Proposition 1

The hypothesis that $\mathbb{K} \supseteq \mathbb{S}_{+}^{V}$ holds cannot be dropped from Theorem 6 . Consider the cone $\mathcal{C}_{V}^{*}$ of completely positive matrices. Now take $V:=\{1, \ldots, n\}$ for some $n \geq 2$ and note that both $\mathbb{1}_{\{0,1\}} \mathbb{1}_{\{0,1\}}^{\top}+\mathbb{1}_{\{2\}} \mathbb{1}_{\{2\}}^{\top}$ and $\mathbb{1}_{\{0,2\}} \mathbb{1}_{\{0,2\}}^{\top}+\mathbb{1}_{\{1\}} \mathbb{1}_{\{1\}}^{\top}$ lie in $\operatorname{Schur}\left(\mathcal{C}_{V}^{*}\right)$, whereas their midpoint does not.

Similar constructions of higher dimensional cones from lower dimensional cones exist in many other areas of mathematics and mathematical sciences. In addition to the obvious Schur complement connection, there are at least two other instances: one in the construction of Siegel Domains (see [24, 47] and the references therein to start), another in convex optimization and analysis in certain recursive quadratic reformulation of optimization problems (see [40, pp. 165-168]). In both of these constructions (which guarantee the convexity of the resulting cone), $x x^{\top}$ corresponds to the bilinear form. A key condition on the bilinear forms in both of these constructions, corresponds to the condition $x x^{\top} \in \mathbb{K}$ in our current context. Indeed, this last condition is equivalent to $\mathbb{K} \supseteq \mathbb{S}_{+}^{V}$.

PSD and Schur liftings of cones are in a sense dual to each other. In the next result, we make this statement a bit clearer by showing a containment relation between theta bodies defined using these two liftings. The relation may be regarded as a form of Weak Duality, and we shall later prove that equality, and hence a form of Strong Duality, holds.

Proposition 7. Let $\mathbb{A} \subseteq \mathbb{S}^{V}$ be a diagonally scaling-invariant polyhedral cone such that $\operatorname{Im}(\operatorname{Diag}) \subseteq \mathbb{A}$. Let $\mathbb{K} \subseteq \mathbb{S}_{+}^{V}$ be a diagonally scaling-invariant closed convex cone such that $\operatorname{Diag}\left(\mathbb{R}_{+}^{V}\right) \subseteq \mathbb{K}$. Then

$$
\operatorname{TH}(\mathbb{A}, \operatorname{Psd}(\mathbb{K})) \subseteq \operatorname{abl}\left(\operatorname{cl}\left(\operatorname{TH}\left(\overline{\mathbb{A}}, \operatorname{Schur}\left(\mathbb{K}^{*}\right)\right)\right)\right) .
$$

Proof. By continuity, it suffices to show that $\langle x, y\rangle \leq 1$ if $x \in \mathrm{TH}(\mathbb{A}, \operatorname{Psd}(\mathbb{K}))$ and $y \in \operatorname{TH}\left(\overline{\mathbb{A}}, \operatorname{Schur}\left(\mathbb{K}^{*}\right)\right)$. Let $x \in \mathrm{TH}(\mathbb{A}, \operatorname{Psd}(\mathbb{K}))$, and let $\hat{X} \in \widehat{\mathrm{TH}}(\mathbb{A}, \operatorname{Psd}(\mathbb{K}))$ such that $x=\operatorname{diag}(X)$ for $X:=\hat{X}[V]$. Let $y \in$ $\operatorname{TH}\left(\overline{\mathbb{A}}, \operatorname{Schur}\left(\mathbb{K}^{*}\right)\right)$, and let $\hat{Y} \in \widehat{\mathrm{TH}}\left(\overline{\mathbb{A}}, \operatorname{Schur}\left(\mathbb{K}^{*}\right)\right)$ such that $y=\operatorname{diag}(Y)$ for $Y:=\hat{Y}[V]$. Write $Y=$ $\operatorname{Diag}(u)-B$ where $B \in \mathbb{A}^{*}$. Since $\mathbb{A}^{*} \subseteq \operatorname{Null}(\operatorname{diag})$, we have $u=y$. Then

$$
\begin{aligned}
0 & \leq\left\langle X, Y-y y^{\top}\right\rangle=\langle X, \operatorname{Diag}(u)-B\rangle-y^{\top} X y=\langle x, y\rangle-\langle X, B\rangle-y^{\top} X y \\
& \leq\langle x, y\rangle-y^{\top}\left(x x^{\top}\right) y=\langle x, y\rangle-\langle x, y\rangle^{2} .
\end{aligned}
$$

Hence, $\langle x, y\rangle \leq 1$.

We can now give an example of a natural theta body defined over the Schur lifting of a cone. Define the weak fractional stable set polytope of a graph $G=(V, E)$ as the polytope

$$
\operatorname{FRAC}(G):=\left\{x \in[0,1]^{V}: x_{i}+x_{j} \leq 1 \forall i j \in E\right\} .
$$

We shall prove that $\operatorname{FRAC}(G)$ is a theta body over the Schur lifting of the convex cone

$$
\mathbb{K}_{2}^{V}:=\left\{X \in \mathbb{S}^{V}: X[e] \succeq 0 \forall e \in\left(\begin{array}{c}
V \\
2
\end{array}\right)\right\} ;
$$

note the similarity with the hypothesis of Corollary 2 . We shall make essential use of Theorem [6 in our proof.

Theorem 8. Let $G=(V, E)$ be a graph such that $|V| \geq 2$. Then

$$
\operatorname{FRAC}(G)=\operatorname{TH}\left(\mathbb{A}_{E, E}, \operatorname{Schur}\left(\mathbb{K}_{2}^{V}\right)\right) .
$$

Proof. We first prove ' $\supseteq$ '. Let $x \in \mathrm{TH}\left(\mathbb{A}_{E, E}, \operatorname{Schur}\left(\mathbb{K}_{2}^{V}\right)\right)$, and let $\hat{X} \in \widehat{\mathrm{TH}}\left(\mathbb{A}_{E, E}, \operatorname{Schur}\left(\mathbb{K}_{2}^{V}\right)\right)$ such that $x=\operatorname{diag}(X)$ for $X:=\hat{X}[V]$. By Theorem[6] we have $x \in[0,1]^{V}$. Let $e=i j \in E$. Set $Y:=X[e]$ and $y:=x \uparrow_{e}$. Then $X \succeq_{\mathbb{K}_{2}^{V}} x x^{\top}$ implies $Y \succeq y y^{\top}$ so

$$
\left[\begin{array}{ccc}
1 & x_{i} & x_{j} \\
x_{i} & x_{i} & 0 \\
x_{j} & 0 & x_{j}
\end{array}\right] \succeq 0 \Longrightarrow\left[\begin{array}{ccc}
1 & -x_{i} & -x_{j} \\
-x_{i} & x_{i} & 0 \\
-x_{j} & 0 & x_{j}
\end{array}\right] \succeq 0 \Longrightarrow 1-x_{i}-x_{j}=\left\langle\left[\begin{array}{ccc}
1 & -x_{i} & -x_{j} \\
-x_{i} & x_{i} & 0 \\
-x_{j} & 0 & x_{j}
\end{array}\right], \mathbb{1}^{\top}\right\rangle \geq 0 .
$$

Thus $x \in \operatorname{FRAC}(G)$, and ' $\supseteq$ ' is proved. 
For the reverse inclusion, it suffices by Theorem 6 to show that $\operatorname{TH}\left(\mathbb{A}_{E, E}, \operatorname{Schur}\left(\mathbb{K}_{2}^{V}\right)\right)$ contains all the extreme points of $\operatorname{FRAC}(G)$. So let $x$ be an extreme point of $\operatorname{FRAC}(G)$. By [43, Theorem 64.7], all coordinates of $x$ lie in $\left\{0, \frac{1}{2}, 1\right\}$. Define

$$
\hat{X}:=\left[\begin{array}{ll}
1 & x^{\top} \\
x & X
\end{array}\right] \in \mathbb{S}^{\{0\} \cup V}
$$

by setting $\operatorname{diag}(X):=x$ and $X_{i j}:=[i j \in \bar{E}]\left[x_{i}+x_{j}>1\right] x_{i} x_{j}$ for every $i j \in\left(\begin{array}{c}V \\ 2\end{array}\right)$. Note that $X \in \mathbb{A}_{E, E} \cap \mathbb{K}_{2}^{V}$ holds, and that $X \succeq_{\mathbb{K}_{2}^{V}} x x^{\top}$ is equivalent to

$$
Y^{i j}:=\left[\begin{array}{ccc}
1 & x_{i} & x_{j} \\
x_{i} & x_{i} & X_{i j} \\
x_{j} & X_{i j} & x_{j}
\end{array}\right] \in \mathbb{S}_{+}^{\{0\} \cup\{i, j\}},
$$

for every $i j \in\left(\begin{array}{c}V \\ 2\end{array}\right)$. So let $i j \in\left(\begin{array}{c}V \\ 2\end{array}\right)$. If $x_{i}+x_{j} \leq 1$, then $X_{i j}=0$ and either $0 \in\left\{x_{i}, x_{j}\right\}$ or $x_{i}=x_{j}=\frac{1}{2}$, so $Y^{i j} \succeq 0$ is easily verified. So assume $x_{i}+x_{j}>1$. Then $i j \in \bar{E}$, so $X_{i j}=x_{i} x_{j}$. If $x_{i}=x_{j}=1$, then $Y^{i j}=\mathbb{1} \mathbb{1}^{\top} \succeq 0$. If $x_{i}=1$ and $x_{j}=\frac{1}{2}$, then

$$
Y^{i j}=\left[\begin{array}{ccc}
1 & 1 & \frac{1}{2} \\
1 & 1 & \frac{1}{2} \\
\frac{1}{2} & \frac{1}{2} & \frac{1}{2}
\end{array}\right]=\mathcal{D}_{\mathbb{1}-e_{2} / 2}\left(\mathbb{1} \mathbb{1}^{\top}+e_{2} e_{2}^{\top}\right) \succeq 0 .
$$

Thus, $\hat{X} \in \operatorname{Schur}\left(\mathbb{K}_{2}^{V}\right)$ and the proof of ' $\subseteq$ ' is complete.

\section{Reformulations of Antiblocking Duality}

We saw in Section 4.1 that the support functions of some theta bodies defined over the PSD lifting of a cone $\mathbb{K} \subseteq \mathbb{S}^{V}$ may be expressed as a conic optimization problem over $\mathbb{K}$. In this section, we shall see that something similar holds for Schur liftings. This shall be essentially a manifestation of antiblocking duality, namely, that $\operatorname{abl}(\operatorname{abl}(\mathscr{C}))=\mathscr{C}$ for every convex corner $\mathscr{C}$; we shall make use of this fact throughout the rest of the paper. At the end of the section, we shall have an expression for $\vartheta_{2}$ from (1.6), and we shall also introduce $\vartheta_{1}$ along the way.

In the next result, we follow the rules set for [23, Eq. (9.3.6)] to interpret the quotient $w_{i} / s_{i}$, with $w_{i}, s_{i} \in \mathbb{R}_{+}$:

if $w_{i}=0$, then we take the fraction $w_{i} / s_{i}$ to be 0 , even if the denominator is 0 ; if $w_{i}>0$

but the denominator is 0 , we take the fraction $w_{i} / s_{i}$ to be $+\infty$.

Proposition 9. Let $\mathscr{C} \subseteq \mathbb{R}^{V}$ be a convex corner. Let $w \in \mathbb{R}_{+}^{V}$. Then

$$
\delta^{*}(w \mid \mathscr{C})=\min _{s \in \operatorname{abl}(\mathscr{C})} \max _{i \in V} \frac{w_{i}}{s_{i}} .
$$

In particular,

$$
\delta^{*}(w \mid \operatorname{abl}(\mathscr{C}))=\min _{x \in \mathscr{C}} \max _{i \in V} \frac{w_{i}}{x_{i}} .
$$

Moreover, all four optimization problems in (5.2) and (5.3) have optimal solutions.

Proof. We may assume that $w \neq 0$. Let us prove ' $\leq$ '. Let $x \in \mathscr{C}$ and $s \in \operatorname{abl}(\mathscr{C})$. We may assume that the $\max$ on the RHS is finite so that, by following the rules from (5.1), we have $W:=\operatorname{supp}(w) \subseteq \operatorname{supp}(s)=: S$. Then

$$
\langle w, x\rangle=\sum_{i \in W} w_{i} x_{i}=\sum_{i \in S} \frac{w_{i}}{s_{i}} s_{i} x_{i} \leq\left(\max _{i \in S} \frac{w_{i}}{s_{i}}\right) \sum_{i \in V} s_{i} x_{i} \leq \max _{i \in V} \frac{w_{i}}{s_{i}}
$$

where (5.1) is only used in the rightmost term. For the reverse inequality, let $\vartheta:=\delta^{*}(w \mid \mathscr{C})>0$. Then $s:=\frac{1}{\vartheta} w \in \operatorname{abl}(\mathscr{C})$. Since $\max _{i \in V} w_{i} / s_{i}=\vartheta$, we find that the RHS of (5.2) is bounded above by $\vartheta=\delta^{*}(w \mid \mathscr{C})$. This proves ' $\geq$ ' in (5.2), as well as attainment for its RHS. Finally, (5.3) follows from (5.2) by antiblocking duality. 
We shall later formulate the parameter $\vartheta_{1}$ (see the discussion in Subsection 1.1) essentially as the optimization problem in the RHS of (5.3) applied to a theta body. In a way, that formulation is unnecessary for the proof of the generalization of (1.4), and it may be further simplified as a line-search, i.e., by a gauge function:

Proposition 10. Let $\mathscr{C} \subseteq \mathbb{R}^{V}$ be a convex corner. Let $w \in \mathbb{R}_{+}^{V}$. Then

$$
\min _{x \in \mathscr{C}} \max _{i \in V} \frac{w_{i}}{x_{i}}=\min \left\{\lambda \in \mathbb{R}_{+}: w \in \lambda \mathscr{C}\right\} .
$$

Moreover, the RHS is attained.

Proof. We may assume that $w \neq 0$. First we show ' $\leq$ '. Let $\lambda \in \mathbb{R}_{+}$such that $w \in \lambda \mathscr{C}$. Then $\lambda>0$ since $w \neq 0$. Set $x:=\frac{1}{\lambda} w \in \mathscr{C}$. Then $w_{i} / x_{i}=\left[w_{i} \neq 0\right] \lambda$ for every $i \in V$, according to the rules from (5.1), so that $\max _{i \in V} w_{i} / x_{i}=\lambda$, whence the LHS of (5.4) is $\leq \lambda$. This proves ' $\leq$ '.

For the reverse inequality, let $x \in \mathscr{C}$ attain the LHS of (5.4), and let $\lambda:=\max _{i \in V} w_{i} / x_{i}$. Since $w \neq 0$, we have $\lambda>0$. It is easy to check that $y:=\frac{1}{\lambda} w$ satisfies $y \leq x$. Since $0 \leq y \leq x \in \mathscr{C}$ and $\mathscr{C}$ is lowercomprehensive, we find that $y \in \mathscr{C}$, i.e., $w \in \lambda \mathscr{C}$. This proves ' $\geq$ ' on (5.4), as well as attainment in its RHS.

The RHS of (5.4) is, by definition, the gauge function $\gamma(w \mid \mathscr{C})$ of $\mathscr{C}$ at $w$, i.e., $\gamma(x \mid \mathscr{C})$ is defined as

$$
\gamma(x \mid \mathscr{C}):=\inf \left\{\mu: \mu \in \mathbb{R}_{+}, x \in \mu \mathscr{C}\right\} \quad \forall x \in \mathbb{R}^{V} .
$$

From Propositions 9 and 10, we recover the fact that

$$
\text { for a convex corner } \mathscr{C} \subseteq \mathbb{R}_{+}^{V} \text {, we have } \delta^{*}(\cdot \mid \operatorname{abl}(\mathscr{C}))=\gamma(\cdot \mid \mathscr{C}) \text { on } \mathbb{R}_{+}^{V} ;
$$

see [41, Theorem 14.5].

A gauge function is oblivious to the upper surface of a set which is "almost" a convex corner:

Proposition 11. Let $\mathscr{C} \subseteq \mathbb{R}_{+}^{V}$ be a lower-comprehensive convex set with nonempty interior. Then

$$
\gamma(w \mid \mathscr{C})=\gamma(w \mid \operatorname{cl}(\mathscr{C})) \quad \forall w \in \mathbb{R}_{+}^{V} .
$$

Proof. The proof of ' $\geq$ ' is obvious. For the reverse inequality, let $w \in \mathbb{R}_{+}^{V}$ and let $\lambda \in \mathbb{R}_{+}$such that $w \in \lambda \operatorname{cl}(\mathscr{C})$. If $\lambda=0$, then $w=0$ and $\gamma(w \mid \mathscr{C})=0=\gamma(w \mid \operatorname{cl}(\mathscr{C}))$, so assume $\lambda>0$. We will show that $w \in(\lambda+\varepsilon) \mathscr{C}$ for every $\varepsilon>0$. Let $\varepsilon>0$. Since $\mathscr{C}$ is lower-comprehensive and has nonempty interior, there exists $M \in \mathbb{R}_{++}$such that $\mathbb{1} / M \in \operatorname{int}(\mathscr{C})$. Thus, for every $\mu \in \mathbb{R}$ such that $0<\mu \leq 1$, we have $\frac{\mu}{M} \mathbb{1}+\frac{1-\mu}{\lambda} w \in \operatorname{int}(\mathscr{C})$. For $\mu:=\varepsilon /(\lambda+\varepsilon)$, this gives $\frac{\varepsilon}{M(\lambda+\varepsilon)} \mathbb{1}+\frac{1}{\lambda+\varepsilon} w \in \operatorname{int}(\mathscr{C})$, and since $\mathscr{C}$ is lowercomprehensive, we get $w \in(\lambda+\varepsilon) \mathscr{C}$. Since $\varepsilon>0$ was arbitrary, this proves ' $\leq$ ' in (5.7).

We are now ready to show how an optimization problem over Schur( $\mathbb{K})$ may sometimes be reduced to an optimization problem over $\mathbb{K}$. We shall use the following simple fact:

$$
\text { if } \mathbb{K} \supseteq \mathbb{S}_{+}^{V} \text {, then }\left[\begin{array}{cc}
1 & x^{\top} \\
x & X
\end{array}\right] \in \operatorname{Schur}(\mathbb{K}) \text { if and only if } X \succeq_{\mathbb{K}} x x^{\top} .
$$

Proposition 12. Let $\mathbb{A} \subseteq \mathbb{S}^{V}$ and $\widehat{\mathbb{K}} \subseteq \mathbb{S}_{\{0\} \cup V}$ be diagonally scaling-invariant closed convex cones such that (2.6) and (2.7) hold. Let $w \in \mathbb{R}_{+}^{V}$. Then

$$
\delta^{*}(w \mid \operatorname{abl}(\operatorname{cl}(\operatorname{TH}(\mathbb{A}, \widehat{\mathbb{K}}))))=\inf \left\{\lambda \in \mathbb{R}_{+}: W \in \mathbb{A}, \operatorname{diag}(W)=\lambda \mathbb{1},\left[\begin{array}{cc}
1 & \sqrt{w}^{\top} \\
\sqrt{w} & W
\end{array}\right] \in \widehat{\mathbb{K}}\right\} .
$$

In particular, if $\mathbb{A}$ is polyhedral, then

$$
\delta^{*}(w \mid \operatorname{abl}(\operatorname{cl}(\operatorname{TH}(\mathbb{A}, \widehat{\mathbb{K}}))))=\inf \left\{\lambda \in \mathbb{R}_{+}: Y \in-\mathbb{A} \cap \operatorname{Null}(\operatorname{diag}),\left[\begin{array}{cc}
1 & \sqrt{w}^{\top} \\
\sqrt{w} & \lambda I-Y
\end{array}\right] \in \widehat{\mathbb{K}}\right\},
$$

and if $\mathbb{K} \subseteq \mathbb{S}^{V}$ is a diagonally scaling-invariant closed convex cone such that $\mathbb{K} \supseteq \mathbb{S}_{+}^{V}$ and $\operatorname{diag}(\mathbb{K}) \subseteq \mathbb{R}_{+}^{V}$ then

$$
\delta^{*}(w \mid \operatorname{abl}(\operatorname{cl}(\operatorname{TH}(\mathbb{A}, \operatorname{Schur}(\mathbb{K})))))=\inf \left\{\lambda: \lambda I \succeq_{\mathbb{K}} Y+\sqrt{w} \sqrt{w}^{\top}, Y \in-\mathbb{A} \cap \operatorname{Null}(\operatorname{diag})\right\} .
$$

Moreover, for each of (5.9), (5.10), and (5.11), if the theta body on the LHS is closed, then the optimization problem on the RHS has an optimal solution. 
Proof. We may assume that $w \neq 0$. From Propositions 1 and 11 and from (5.6), we have

$$
\delta^{*}(w \mid \operatorname{abl}(\operatorname{cl}(\operatorname{TH}(\mathbb{A}, \widehat{\mathbb{K}}))))=\gamma(w \mid \operatorname{TH}(\mathbb{A}, \widehat{\mathbb{K}}))=\inf \Lambda, \quad \text { where } \Lambda:=\left\{\lambda \in \mathbb{R}_{++}: w \in \lambda \operatorname{TH}(\mathbb{A}, \widehat{\mathbb{K}})\right\}
$$

Note that $\Lambda$ is convex, unbounded above, and bounded away from zero since the LHS of (5.12) is positive by Proposition 1. Moreover, $\Lambda$ is closed if $\mathrm{TH}(\mathbb{A}, \widehat{\mathbb{K}})$ is closed by the attainment statement in Proposition 10 . We may reformulate the set $\Lambda$ as

$$
\begin{aligned}
\Lambda & =\left\{\lambda \in \mathbb{R}_{++}: W \in \mathbb{A}, \operatorname{diag}(W)=\frac{1}{\lambda} w,\left[\begin{array}{cc}
1 & \frac{1}{\lambda} w^{\top} \\
\frac{1}{\lambda} w & W
\end{array}\right] \in \widehat{\mathbb{K}}\right\} \\
& =\left\{\lambda \in \mathbb{R}_{++}: X \in \mathbb{A}, \operatorname{diag}(X)=\lambda \mathbb{1}_{\operatorname{supp}(w)},\left[\begin{array}{cc}
1 & \sqrt{w}^{\top} \\
\sqrt{w} & X
\end{array}\right] \in \widehat{\mathbb{K}}\right\} \\
& =\left\{\lambda \in \mathbb{R}_{++}: X \in \mathbb{A}, \operatorname{diag}(X)=\lambda \mathbb{1},\left[\begin{array}{cc}
1 & \sqrt{w}^{\top} \\
\sqrt{w} & X
\end{array}\right] \in \widehat{\mathbb{K}}\right\},
\end{aligned}
$$

where we used the diagonal scaling invariance of $\mathbb{A}$ and $\widehat{\mathbb{K}}$ and the change of variable

$$
\hat{X}=\mathcal{D}_{1 \oplus \lambda w^{-1 / 2}}\left(\left[\begin{array}{cc}
1 & \frac{1}{\lambda} w^{\top} \\
\frac{1}{\lambda} w & W
\end{array}\right]\right), \quad \text { with }\left[w^{-1 / 2}\right]_{i}:= \begin{cases}w_{i}^{-1 / 2} & \text { if } w_{i}>0 \\
1 & \text { otherwise }\end{cases}
$$

for the second equation, and the diagonal scaling invariance of $\mathbb{A}$ and $\widehat{\mathbb{K}}$ and assumptions (2.6) and (2.7a) for the last equation. To prove (5.9), it now suffices to show that relaxing the constraint $\lambda \in \mathbb{R}_{++}$to $\lambda \in \mathbb{R}_{+}$ in the RHS of (5.13) does not change the set. If it did, the relaxed set, which is convex, would contain 0 , and so $\Lambda$ would not be bounded away from zero, a contradiction.

Suppose that $\mathbb{A}$ is polyhedral. It is easy to check that $\mathbb{A} \cap \operatorname{diag}^{-1}(\lambda \mathbb{1})=\lambda I-(-\mathbb{A} \cap \operatorname{Null}(\operatorname{diag}))$; the inclusion ' $\supseteq$ ' is obvious, whereas the reverse inclusion follows from Proposition 3 Thus, (5.10) follows. Equation (5.11) follows from (5.10) and Theorem 6, using the equivalence (5.8). The constraint $\lambda \in \mathbb{R}_{+}$ may be dropped since $\operatorname{diag}(\mathbb{K}) \subseteq \mathbb{R}_{+}^{V}$. In all cases, attainment if the theta body is closed follows from the closedness of $\Lambda$.

\section{A Plethora of Theta Functions}

We have now introduced all formulations of the parameters $\vartheta_{i}$ 's from (1.6) and we are ready to prove that they are all equal. Let $\mathbb{A} \subseteq \mathbb{S}^{V}$ be a diagonally scaling-invariant polyhedral cone such that $\operatorname{Im}(\operatorname{Diag}) \subseteq \mathbb{A}$. Let $\mathbb{K} \subseteq \mathbb{S}_{+}^{V}$ be a diagonally scaling-invariant closed convex cone such that $\operatorname{Diag}\left(\mathbb{R}_{+}^{V}\right) \subseteq \mathbb{K}$ and $\operatorname{int}(\mathbb{K}) \neq \varnothing$. For each $w \in \mathbb{R}_{+}^{V}$, define:

$$
\begin{gathered}
\vartheta(\mathbb{A}, \mathbb{K} ; w):=\delta^{*}\left(w \mid \operatorname{abl}\left(\operatorname{cl}\left(\operatorname{TH}\left(\overline{\mathbb{A}}, \operatorname{Schur}\left(\mathbb{K}^{*}\right)\right)\right)\right),\right. \\
\vartheta_{1}(\mathbb{A}, \mathbb{K} ; w):=\inf \left\{\max _{i \in V} \frac{w_{i}}{x_{i}}: x \in \operatorname{cl}\left(\operatorname{TH}\left(\overline{\mathbb{A}}, \operatorname{Schur}\left(\mathbb{K}^{*}\right)\right)\right)\right\}, \\
\vartheta_{2}(\mathbb{A}, \mathbb{K} ; w):=\inf \left\{\lambda: \lambda I \succeq_{\mathbb{K}^{*}} Y+\sqrt{w} \sqrt{w}^{\top}, Y \in-\overline{\mathbb{A}} \cap \operatorname{Null}(\operatorname{diag})\right\}, \\
\vartheta_{3}(\mathbb{A}, \mathbb{K} ; w):=\sup \left\{\sqrt{w}^{\top} X \sqrt{w}: \operatorname{Tr}(X)=1, X \in \mathbb{K}, X \in \mathbb{A}\right\}, \\
\vartheta_{4}(\mathbb{A}, \mathbb{K} ; w):=\delta^{*}(w \mid \operatorname{TH}(\mathbb{A}, \operatorname{Psd}(\mathbb{K}))) .
\end{gathered}
$$

Here, the objective function for $\vartheta_{1}(\mathbb{A}, \mathbb{K} ; w)$ is evaluated according to the arithmetic rules from (5.1). For concreteness, we shall finally define the Lovász theta number and the variants $\vartheta^{\prime}$ and $\vartheta^{+}$as special cases of the above parameters. Let $G=(V, E)$ be a graph. For each $w \in \mathbb{R}_{+}^{V}$, define

$$
\begin{gathered}
\vartheta(G ; w):=\vartheta\left(\mathbb{A}_{E, E}, \mathbb{S}_{+}^{V} ; w\right), \\
\vartheta^{\prime}(G ; w):=\vartheta\left(\mathbb{A}_{E \cup \bar{E}, E}, \mathbb{S}_{+}^{V} ; w\right), \\
\vartheta^{+}(G ; w):=\vartheta\left(\mathbb{A}_{\varnothing, E}, \mathbb{S}_{+}^{V} ; w\right) .
\end{gathered}
$$


Theorem 13. Let $\mathbb{A} \subseteq \mathbb{S}^{V}$ be a diagonally scaling-invariant polyhedral cone such that $\operatorname{Im}(\operatorname{Diag}) \subseteq \mathbb{A}$. Let $\mathbb{K} \subseteq \mathbb{S}_{+}^{V}$ be a diagonally scaling-invariant closed convex cone such that $\operatorname{Diag}\left(\mathbb{R}_{+}^{V}\right) \subseteq \mathbb{K}$ and $\operatorname{int}(\mathbb{K}) \neq \varnothing$. Let $w \in \mathbb{R}_{+}^{V}$. Then

$$
\vartheta(\mathbb{A}, \mathbb{K} ; w)=\vartheta_{1}(\mathbb{A}, \mathbb{K} ; w)=\vartheta_{2}(\mathbb{A}, \mathbb{K} ; w)=\vartheta_{3}(\mathbb{A}, \mathbb{K} ; w)=\vartheta_{4}(\mathbb{A}, \mathbb{K} ; w) .
$$

Moreover, all optimization problems in (6.2) have optimal solutions except possibly for $\vartheta_{2}$, which has an optimal solution if $\operatorname{TH}\left(\overline{\mathbb{A}}, \operatorname{Schur}\left(\mathbb{K}^{*}\right)\right)$ is closed. Furthermore,

$$
\operatorname{abl}\left(\operatorname{cl}\left(\operatorname{TH}\left(\overline{\mathbb{A}}, \operatorname{Schur}\left(\mathbb{K}^{*}\right)\right)\right)\right)=\operatorname{TH}(\mathbb{A}, \operatorname{Psd}(\mathbb{K})) .
$$

Proof. The optimization problems that define $\vartheta_{2}(\mathbb{A}, \mathbb{K} ; w)$ and $\vartheta_{3}(\mathbb{A}, \mathbb{K} ; w)$ form a primal-dual pair of conic optimization problems; this follows from the polyhedrality of $\mathbb{A}$ and from $\operatorname{Im}(\operatorname{Diag}) \subseteq \mathbb{A}$. Thus, the equation $\vartheta_{2}(\mathbb{A}, \mathbb{K} ; w)=\vartheta_{3}(\mathbb{A}, \mathbb{K} ; w)$ follows by Conic Programming Strong Duality; see, e.g., [6] or [8], Theorem 1.1]. Although the conic formulation for $\vartheta_{3}(\mathbb{A}, \mathbb{K} ; w)$ need not have a Slater point, the assumptions that $\mathbb{A}$ is polyhedral and $\mathbb{K}^{*} \supseteq \mathbb{S}_{+}^{V}$ show that the optimization problem defining $\vartheta_{2}(\mathbb{A}, \mathbb{K} ; w)$ has a restricted Slater point. Equation (6.2) follows from $\vartheta_{2}(\mathbb{A}, \mathbb{K} ; w)=\vartheta_{3}(\mathbb{A}, \mathbb{K} ; w)$, Propositions 9 and 12 , and Theorem 5 since $\operatorname{cl}\left(\operatorname{TH}\left(\overline{\mathbb{A}}, \operatorname{Schur}\left(\mathbb{K}^{*}\right)\right)\right)$ is a convex corner by Theorem [6 Existence of optimal solutions follows from the corresponding statements in the previous results. Now (6.3) follows from conjugate duality applied to $\vartheta(\mathbb{A}, \mathbb{K} ; w)=\vartheta_{4}(\mathbb{A}, \mathbb{K} ; w)$ for every $w \in \mathbb{R}_{+}^{V}$.

Theorem 13 implies (1.4a) and (1.4b) using the descriptions (2.5) for every graph $G$. Note also that we could have mimicked the proof of the chain (1.6) as in [23] and [30]; the proof that $\vartheta_{4}(\mathbb{A}, \mathbb{K} ; w) \leq \vartheta(\mathbb{A}, \mathbb{K} ; w)$ follows from Proposition 7.

In the context of Theorem 13, the support functions of the two theta bodies that appear in (6.3) are gauges polar to each other; see [41, §15] and recall the definition of gauge from (5.5). The corresponding polar inequality (that is, the corresponding Cauchy-Schwarz inequality) for these gauges is stated next; compare with [14, Proposition 8 and Theorem 18]. For each permutation $\sigma$ on $V$, define the linear map $P(\sigma): \mathbb{R}^{V} \rightarrow \mathbb{R}^{V}$ as the linear extension of the map $e_{i} \in \mathbb{R}^{V} \mapsto e_{\sigma(i)}$. For each $L \in \mathbb{R}^{V \times V}$, define the congruence map Congr $_{L}: \mathbb{R}^{V \times V} \rightarrow \mathbb{R}^{V \times V}$ as

$$
\operatorname{Congr}_{L}(X):=L X L^{\top} \quad \forall X \in \mathbb{R}^{V \times V} .
$$

Corollary 14. Let $\mathbb{A} \subseteq \mathbb{S}^{V}$ be a diagonally scaling-invariant polyhedral cone such that $\operatorname{Im}(\operatorname{Diag}) \subseteq \mathbb{A}$. Let $\mathbb{K} \subseteq \mathbb{S}_{+}^{V}$ be a diagonally scaling-invariant closed convex cone such that $\operatorname{Diag}\left(\mathbb{R}_{+}^{V}\right) \subseteq \mathbb{K}$ and $\operatorname{int}(\mathbb{K}) \neq \varnothing$. If $w, \bar{w} \in \mathbb{R}_{+}^{V}$, then

$$
\langle w, \bar{w}\rangle \leq \delta^{*}(w \mid \operatorname{TH}(\mathbb{A}, \operatorname{Psd}(\mathbb{K}))) \cdot \delta^{*}\left(\bar{w} \mid \operatorname{cl}\left(\operatorname{TH}\left(\overline{\mathbb{A}}, \operatorname{Schur}\left(\mathbb{K}^{*}\right)\right)\right)\right) .
$$

Moreover, if there exists a transitive permutation group $\Gamma$ on $V$ such that

$$
\left\{\operatorname{Congr}_{P(\sigma)}: \sigma \in \Gamma\right\} \subseteq \operatorname{Aut}(\mathbb{A}) \cap \operatorname{Aut}(\mathbb{K}),
$$

then

$$
|V|=\delta^{*}(\mathbb{1} \mid \operatorname{TH}(\mathbb{A}, \operatorname{Psd}(\mathbb{K}))) \cdot \delta^{*}\left(\mathbb{1} \mid \operatorname{cl}\left(\operatorname{TH}\left(\overline{\mathbb{A}}, \operatorname{Schur}\left(\mathbb{K}^{*}\right)\right)\right)\right) .
$$

Proof. By Theorem [6, we know that $\operatorname{cl}\left(\mathrm{TH}\left(\overline{\mathbb{A}}, \operatorname{Schur}\left(\mathbb{K}^{*}\right)\right)\right)$ is a convex corner. By (5.6) and Theorem 13 , the gauge function $\gamma\left(\cdot \mid \operatorname{cl}\left(\operatorname{TH}\left(\overline{\mathbb{A}}, \operatorname{Schur}\left(\mathbb{K}^{*}\right)\right)\right)\right)$ is the support function $\delta^{*}(\cdot \mid \operatorname{TH}(\mathbb{A}, \operatorname{Psd}(\mathbb{K})))$. Hence, the support functions $\delta^{*}\left(\cdot \mid \operatorname{cl}\left(\operatorname{TH}\left(\overline{\mathbb{A}}, \operatorname{Schur}\left(\mathbb{K}^{*}\right)\right)\right)\right)$ and $\delta^{*}(\cdot \mid \operatorname{TH}(\mathbb{A}, \operatorname{Psd}(\mathbb{K})))$ are gauges polar to each other (when restricted to $\mathbb{R}_{+}^{V}$ ); see [11, Corollary 15.1.2]. Now (6.5) follows immediately.

Next, we prove that ' $\geq$ ' holds in (6.7) if $w=\bar{w}=\mathbb{1}$ and (6.6) holds. Assume the latter, and let $\widehat{\Gamma}$ denote the permutation group $\widehat{\Gamma}:=\left\{\hat{\sigma}: \hat{\sigma}(0)=0,\left.\hat{\sigma}\right|_{V} \in \Gamma\right\}$ on $\{0\} \cup V$. It is clear that $\left\{\operatorname{Congr}_{P(\hat{\sigma})}: \hat{\sigma} \in \widehat{\Gamma}\right\} \subseteq$ $\operatorname{Aut}(\widehat{\mathbb{K}})$ for each $\widehat{\mathbb{K}} \in\left\{\operatorname{Psd}(\mathbb{K}), \operatorname{Schur}\left(\mathbb{K}^{*}\right)\right\}$. Together with $\left\{\operatorname{Congr}_{P(\sigma)}: \sigma \in \Gamma\right\} \subseteq \operatorname{Aut}(\mathbb{A})$, this yields $\left\{\operatorname{Congr}_{P(\hat{\sigma})}: \hat{\sigma} \in \widehat{\Gamma}\right\} \subseteq \operatorname{Aut}(\widehat{\mathscr{C}})$ for each $\widehat{\mathscr{C}} \in\left\{\widehat{\mathrm{TH}}(\mathbb{A}, \operatorname{Psd}(\mathbb{K})), \widehat{\mathrm{TH}}\left(\widehat{\mathbb{A}}, \operatorname{Schur}\left(\mathbb{K}^{*}\right)\right)\right\}$, whence

$$
\{P(\sigma): \sigma \in \Gamma\} \subseteq \operatorname{Aut}(\mathscr{C}) \quad \forall \mathscr{C} \in\left\{\operatorname{TH}(\mathbb{A}, \operatorname{Psd}(\mathbb{K})), \operatorname{TH}\left(\overline{\mathbb{A}}, \operatorname{Schur}\left(\mathbb{K}^{*}\right)\right)\right\} .
$$

Thus, each support function on the RHS of (6.7) is attained by a fixed point of the Reynolds operator

$$
x \in \mathbb{R}^{V} \mapsto \frac{1}{|\Gamma|} \sum_{\sigma \in \Gamma} P(\sigma) x .
$$


Since $\Gamma$ acts transitively on $V$, there exist $\mu, \nu \in \mathbb{R}$ such that $\mu \mathbb{1}$ attains $\delta^{*}(\mathbb{1} \mid \operatorname{TH}(\mathbb{A}, \operatorname{Psd}(\mathbb{K})))$ and $\nu \mathbb{1}$ attains $\delta^{*}\left(\mathbb{1} \mid \operatorname{cl}\left(\operatorname{TH}\left(\overline{\mathbb{A}}, \operatorname{Schur}\left(\mathbb{K}^{*}\right)\right)\right)\right)$. By (6.3) from Theorem [13, we get $\langle\mu \mathbb{1}, \nu \mathbb{1}\rangle \leq 1$ so $\mu \nu|V| \leq 1$. Thus,

$$
\delta^{*}(\mathbb{1} \mid \operatorname{TH}(\mathbb{A}, \operatorname{Psd}(\mathbb{K}))) \cdot \delta^{*}\left(\mathbb{1} \mid \operatorname{cl}\left(\operatorname{TH}\left(\overline{\mathbb{A}}, \operatorname{Schur}\left(\mathbb{K}^{*}\right)\right)\right)\right)=\langle\mathbb{1}, \mu \mathbb{1}\rangle\langle\mathbb{1}, \nu \mathbb{1}\rangle=\mu \nu|V|^{2} \leq|V| .
$$

\section{Theta Bodies over the Copositive and Completely Positive Cones}

In this section, we show that the stable set polytope of a graph and one of its classical fractional relaxations are theta bodies. The key result we use to prove this is a completely positive formulation for the stability number of a graph, due to de Klerk and Pasechnik [13. As a consequence of the antiblocker duality relation from Theorem [13, we shall derive a weighted generalization of a copositive formulation for the fractional chromatic number of a graph, due to Dukanovic and Rendl [14].

Let $G=(V, E)$ be a graph. For each $w \in \mathbb{R}_{+}^{V}$, we set

$$
\alpha(G ; w):=\delta^{*}(w \mid \operatorname{STAB}(G)) .
$$

Recall that the stable set polytope $\operatorname{STAB}(G)$ was defined as the convex hull of $\left\{\mathbb{1}_{S}: S \subseteq V\right.$ stable in $\left.G\right\}$, that $\mathcal{C}_{V}$ denotes the cone of copositive matrices, and that $\mathcal{C}_{V}^{*}$ is the cone of completely positive matrices. The key argument in the proof of the next result comes from [13, Theorem 2.2]:

Proposition 15. If $G=(V, E)$ is a graph, then

$$
\operatorname{TH}\left(\mathbb{A}_{E, E}, \operatorname{Psd}\left(\mathcal{C}_{V}^{*}\right)\right)=\operatorname{STAB}(G) .
$$

Proof. To prove ' $\supseteq$ ', note that, if $S \subseteq V$ is a stable set of $G$, then $\left(1 \oplus \mathbb{1}_{S}\right)\left(1 \oplus \mathbb{1}_{S}\right)^{\top} \in \widehat{\mathrm{TH}}\left(\mathbb{A}_{E, E}, \operatorname{Psd}\left(\mathcal{C}_{V}^{*}\right)\right)$, whence $\mathbb{1}_{S} \in \mathrm{TH}\left(\mathbb{A}_{E, E}, \operatorname{Psd}\left(\mathcal{C}_{V}^{*}\right)\right)$. For the reverse inclusion it suffices by conjugate duality and Corollary 2 to show that, for $w \in \mathbb{R}_{+}^{V}$, we have $\alpha(G ; w) \geq \delta^{*}\left(w \mid \operatorname{TH}\left(\mathbb{A}_{E, E}, \operatorname{Psd}\left(\mathcal{C}_{V}^{*}\right)\right)\right)$. Thus, it suffices by Theorem 5 to show that, for $w \in \mathbb{R}_{+}^{V}$, we have

$$
\alpha(G ; w) \geq \max \left\{\sqrt{w}^{\top} X \sqrt{w}: \operatorname{Tr}(X)=1, X \in \mathcal{C}_{V}^{*}, X \in \mathbb{A}_{E, E}\right\} .
$$

Let $w \in \mathbb{R}_{+}^{V}$. We may assume that $w \neq 0$. The extreme rays of the cone $\mathcal{C}_{V}^{*} \cap \mathbb{A}_{E, E}$ are of the form $x x^{\top}$ with $x \in \mathbb{R}_{+}^{V}$ and $\operatorname{supp}(x)$ stable in $G$. So there exists an optimal solution for the RHS of (17.3) of the form $\bar{x} \bar{x}^{\top}$ for some $\bar{x} \in \mathbb{R}_{+}^{V}$ such that $\|\bar{x}\|^{2}=\operatorname{Tr}\left(\bar{x} \bar{x}^{\top}\right)=1$ and $\operatorname{supp}(\bar{x})$ is a stable set in $G$. In fact, for any $y \in \mathbb{R}_{+}^{V}$ such that $\|y\|^{2}=1$ and $\operatorname{supp}(y) \subseteq \operatorname{supp}(\bar{x})$, the point $y y^{\top}$ is feasible in the RHS of (7.3) with objective value $\langle\sqrt{w}, y\rangle^{2}$ whence the RHS of (17.3) is equal to $\max \left\{\langle\sqrt{w}, y\rangle^{2}: y \in \mathbb{R}_{+}^{V},\|y\|^{2}=1, \operatorname{supp}(y) \subseteq \operatorname{supp}(\bar{x})\right\}$. The optimality conditions for this optimization problem (i.e., Cauchy-Schwarz) show that an optimal solution is given by $\bar{y}:=\frac{\sqrt{u}}{\|\sqrt{u}\|}$ where $u:=w \odot \mathbb{1}_{\operatorname{supp}(\bar{x})}$, and its objective value is

$$
\frac{\langle\sqrt{w}, \sqrt{u}\rangle^{2}}{\|\sqrt{u}\|^{2}}=\frac{\langle\sqrt{u}, \sqrt{u}\rangle^{2}}{\|\sqrt{u}\|^{2}}=\|\sqrt{u}\|^{2}=\left\langle w, \mathbb{1}_{\operatorname{supp}(\bar{x})}\right\rangle .
$$

Since $\operatorname{supp}(\bar{x})$ is stable, this concludes our proof of (7.3).

Let $G=(V, E)$ be a graph. The fractional stable set polytope of $G$ is defined as

$$
\operatorname{QSTAB}(G):=\left\{x \in \mathbb{R}_{+}^{V}:\left\langle\mathbb{1}_{K}, x\right\rangle \leq 1 \text { for every clique } K \text { of } G\right\} .
$$

Note that

$$
\operatorname{QSTAB}(G)=\operatorname{abl}(\operatorname{STAB}(\bar{G})) .
$$

For $w \in \mathbb{R}_{+}^{V}$, the fractional chromatic number of $G$ is

$$
\chi^{*}(G ; w):=\delta^{*}(w \mid \operatorname{QSTAB}(\bar{G})) .
$$

Proposition 15 yields immediately a weighted generalization of [14, Corollary 5]:

Corollary 16. Let $G=(V, E)$ be a graph. Let $w \in \mathbb{R}_{+}^{V}$. Then

$$
\chi^{*}(G ; w)=\min \left\{\lambda: Y \in \mathbb{A} \frac{\perp}{E}, \bar{E},\left[\begin{array}{cc}
1 & \sqrt{w}^{\top} \\
\sqrt{w} & \lambda I-Y
\end{array}\right] \in \operatorname{Psd}\left(\mathcal{C}_{V}^{*}\right)\right\} .
$$


Proof. By Proposition 15] and (5.10) from Proposition 12, we have

$$
\begin{aligned}
\chi^{*}(G ; w) & =\delta^{*}(w \mid \operatorname{QSTAB}(\bar{G}))=\delta^{*}(w \mid \operatorname{abl}(\operatorname{STAB}(G)))=\delta^{*}\left(w \mid \operatorname{abl}\left(\operatorname{TH}\left(\mathbb{A}_{E, E}, \operatorname{Psd}\left(\mathcal{C}_{V}^{*}\right)\right)\right)\right) \\
& =\min \left\{\lambda \in \mathbb{R}_{+}: Y \in-\mathbb{A}_{E, E} \cap \operatorname{Null}(\operatorname{diag}),\left[\begin{array}{cc}
1 & \sqrt{w}^{\top} \\
\sqrt{w} & \lambda I-Y
\end{array}\right] \in \operatorname{Psd}\left(\mathcal{C}_{V}^{*}\right)\right\} \\
& =\min \left\{\lambda: Y \in \mathbb{A}_{\bar{E}}^{\perp}, \bar{E},\left[\begin{array}{cc}
1 & \sqrt{w}^{\top} \\
\sqrt{w} & \lambda I-Y
\end{array}\right] \in \operatorname{Psd}\left(\mathcal{C}_{V}^{*}\right)\right\} .
\end{aligned}
$$

The constraint $\lambda \in \mathbb{R}_{+}$may be dropped $\operatorname{since} \operatorname{diag}\left(\mathcal{C}_{V}^{*}\right) \subseteq \mathbb{R}_{+}^{V}$.

By the antiblocker relation from Theorem 13, we know that $\operatorname{QSTAB}(G)$ is the closure of a theta body. Unlike in the cases presented so far, the fact that the latter theta body is actually closed does not follow from our previous results. Thus, we proceed to prove its closedness separately. We shall use an argument from [18, Theorem 5] (more specifically, in the proof of (17.14) below). We denote the maximum norm by $\|\cdot\|_{\infty}$.

Theorem 17. Let $\mathbb{A} \subseteq \mathbb{S}^{V}$ be a diagonally scaling-invariant polyhedral cone such that $\operatorname{Im}(\operatorname{Diag}) \subseteq \mathbb{A}$. Then

$$
\operatorname{TH}\left(\mathbb{A}, \operatorname{Schur}\left(\mathcal{C}_{V}\right)\right)=\left\{\operatorname{diag}(\hat{X}[V]): \hat{X} \in \widehat{\mathrm{TH}}\left(\mathbb{A}, \operatorname{Schur}\left(\mathcal{C}_{V}\right)\right),\|\hat{X}\|_{\infty} \leq 1\right\} .
$$

Consequently, $\operatorname{TH}\left(\mathbb{A}, \operatorname{Schur}\left(\mathcal{C}_{V}\right)\right)$ is a convex corner contained in $[0,1]^{V}$.

Proof. The inclusion ' $\supseteq$ ' in (7.8) is trivial. For the reverse inclusion, let $x \in \operatorname{TH}\left(\mathbb{A}, \operatorname{Schur}\left(\mathcal{C}_{V}\right)\right)$, and let $\hat{Y} \in \widehat{\mathrm{TH}}\left(\mathbb{A}, \operatorname{Schur}\left(\mathcal{C}_{V}\right)\right)$ such that $x=\operatorname{diag}(Y)$ for $Y:=\hat{Y}[V]$. We shall use (5.8) with $\mathbb{K}=\mathcal{C}_{V}$ throughout the proof without further mention. Note that $Y-x x^{\top} \in \mathcal{C}_{V}$ implies that $x-(x \odot x)=\operatorname{diag}\left(Y-x x^{\top}\right) \geq 0$ so

$$
x \in[0,1]^{V} .
$$

Let us prove that

we may assume that $Y \in \mathbb{S}_{\geq 0}^{V}$ and $Y=Y[\operatorname{supp}(x)] \oplus 0$.

Indeed, the principal submatrix $Y=\hat{Y}[V]$ from $\hat{Y}$ may possibly be replaced with

$$
Y-2 \sum\left\{\left[Y_{i j}<0\right] Y_{i j} \operatorname{Sym}\left(e_{i} e_{j}^{\top}\right): i j \in\left(\begin{array}{c}
V \\
2
\end{array}\right)\right\}
$$

(using the notation Sym from (3.1)) without affecting the relations $\hat{Y}[V] \in \mathbb{A}$ or $\hat{Y}[V] \succeq_{\mathcal{C}_{V}} x x^{\top}$, by Proposition 3 and the trivial fact that $\mathcal{C}_{V}+\mathbb{S}_{\geq 0}^{V}=\mathcal{C}_{V}$. Clearly, for $S:=\operatorname{supp}(x)$ and $\bar{x}:=x \uparrow_{S}$, we have $Y[S] \succeq \mathcal{C}_{S} \bar{x} \bar{x}^{\top}$. Thus, by possibly replacing $\hat{Y}[V]$ with $\hat{Y}[S] \oplus 0$ in $\hat{Y}$, we shall have $Y=Y[\operatorname{supp}(x)] \oplus 0$, and the proof of (7.10) is complete. Thus, by possibly restricting our attention to the index set $\operatorname{supp}(x)$,

$$
\text { we may assume that } \operatorname{supp}(x)=V \text {. }
$$

Write $D:=\operatorname{Diag}(x)$ and $B:=Y-D$. Let $G=(V, E)$ be the graph defined by $E:=\left\{i j \in\left(\begin{array}{c}V \\ 2\end{array}\right): B_{i j}>0\right\}$. Define $A \in \mathbb{A} \cap \operatorname{Null}(\operatorname{diag})$ by setting $A_{i j}:=\frac{1}{2}[i j \in E]\left(1 / x_{i}+1 / x_{j}\right)$ for each $i j \in\left(\begin{array}{c}V \\ 2\end{array}\right)$, where we used Proposition 3 to prove membership of $A$ in $\mathbb{A}$. We claim that

$$
D^{-1}+A-\mathbb{1}^{\top} \in \mathcal{C}_{V}
$$

We shall need to consider the following optimization problem in our proof:

$$
\min \left\{h^{\top}\left(D^{-1}+A\right) h: h \in \mathbb{R}_{+}^{V},\langle\mathbb{1}, h\rangle=1\right\} .
$$

Let us show that

there exists an optimal solution $\bar{h}$ for (7.13) whose support is a stable set in $G$.

Indeed, let $\bar{h}$ be an optimal solution for (7.13) with minimal support. Note that an optimal solution exists by continuity and compactness. Suppose that $i j \subseteq \operatorname{supp}(\bar{h})$ for some $i j \in E$. For each $t \in \mathbb{R}$, define $h_{t}:=\bar{h}+t\left(e_{i}-e_{j}\right)$, and note that $h_{t}$ is feasible for (7.13) whenever $t \in\left[-\bar{h}_{i}, \bar{h}_{j}\right]$. The objective value of $h_{t}$ in (7.13) is, $h_{t}^{\top}\left(D^{-1}+A\right) h_{t}=\bar{h}^{\top}\left(D^{-1}+A\right) \bar{h}+2 t\left(e_{i}-e_{j}\right)^{\top}\left(D^{-1}+A\right) \bar{h}=\bar{h}^{\top}\left(D^{-1}+A\right) \bar{h}$, where the final equation follows from the optimality of $\bar{h}=h_{0}$. Since $h_{\bar{t}}$ is feasible in (7.13) for $\bar{t}:=\bar{h}_{j}$ and $\operatorname{supp}\left(h_{\bar{t}}\right) \subsetneq \operatorname{supp}(\bar{h})$, the proof of (7.14) is complete. 
It follows from (7.14) that $\bar{h}^{\top} A \bar{h}=0$ and $\bar{h}^{\top} D^{-1} B D^{-1} \bar{h}=0$. Thus, since $D^{-1} Y D^{-1} \succeq_{\mathcal{C}_{V}} D^{-1} x x^{\top} D^{-1}$ by the diagonal scaling invariance of $\mathcal{C}_{V}$, we get

$$
\begin{aligned}
\bar{h}^{\top}\left(D^{-1}+A\right) \bar{h} & =\bar{h}^{\top} D^{-1} \bar{h}=\bar{h}^{\top}\left(D^{-1} D D^{-1}\right) \bar{h}=\bar{h}^{\top}\left(D^{-1}(D+B) D^{-1}\right) \bar{h} \\
& \geq \bar{h}^{\top} D^{-1} x x^{\top} D^{-1} \bar{h}=\bar{h}^{\top} \mathbb{1} \mathbb{1}^{\top} \bar{h}=1 .
\end{aligned}
$$

Thus, $\min \left\{h^{\top}\left(D^{-1}+A-\mathbb{1}^{\top}\right) h: h \in \mathbb{R}_{+}^{V}, \mathbb{1}^{\top} h=1\right\} \geq 0$ and (7.12) is proved. Set $X:=\mathcal{D}_{x}\left(D^{-1}+A\right)$. Then (7.12) implies $X \succeq_{\mathcal{C}_{V}} \mathcal{D}_{x}\left(\mathbb{1} \mathbb{1}^{\top}\right)=x x^{\top}$. Moreover, $\operatorname{diag}(X)=x$ and, for $i j \in E$, we have

$$
X_{i j}=\left[\mathcal{D}_{x}(A)\right]_{i j}=\frac{x_{i} x_{j}}{2}\left(\frac{1}{x_{i}}+\frac{1}{x_{j}}\right)=\frac{x_{j}+x_{i}}{2} \leq 1
$$

by (7.9). Since $X_{i j}=0$ for $i j \in \bar{E}$, it follows that

$$
\hat{X}:=\left[\begin{array}{cc}
1 & x^{\top} \\
x & X
\end{array}\right] \in \widehat{\mathrm{TH}}\left(\mathbb{A}, \operatorname{Schur}\left(\mathcal{C}_{V}\right)\right)
$$

and $\|\hat{X}\|_{\infty} \leq 1$. This completes the proof of (7.8). It follows that the set $\operatorname{TH}\left(\mathbb{A}, \operatorname{Schur}\left(\mathcal{C}_{V}\right)\right)$ is closed, since it is described by (7.8) as the linear image of a compact set. Thus, $\operatorname{TH}\left(\mathbb{A}, \operatorname{Schur}\left(\mathcal{C}_{V}\right)\right)$ is a convex corner by Theorem 6 .

Corollary 18. Let $G=(V, E)$ be a graph. Then

$$
\operatorname{QSTAB}(G)=\operatorname{TH}\left(\mathbb{A}_{E, E}, \operatorname{Schur}\left(\mathcal{C}_{V}\right)\right) .
$$

In particular, for every $w \in \mathbb{R}_{+}^{V}$, we have

$$
\chi^{*}(G ; w)=\max \left\{\langle w, x\rangle: X \in \mathbb{A}_{\bar{E}, \bar{E}}, \operatorname{diag}(X)=x, X \succeq_{\mathcal{C}_{V}} x x^{\top}\right\} .
$$

Proof. We know that $\operatorname{abl}\left(\operatorname{TH}\left(\mathbb{A}_{E, E}, \operatorname{Schur}\left(\mathcal{C}_{V}\right)\right)\right)=\operatorname{TH}\left(\mathbb{A}_{\bar{E}, \bar{E}}, \operatorname{Psd}\left(\mathcal{C}_{V}^{*}\right)\right)=\operatorname{STAB}(\bar{G})$ by Theorems 13 and 17 and Proposition 15. Thus, (7.15) follows from antiblocking duality. Now (7.16) follows from (7.15) and (5.8) since, for each $w \in \mathbb{R}_{+}^{V}$, we have $\chi^{*}(G ; w)=\delta^{*}(w \mid \operatorname{QSTAB}(\bar{G}))=\delta^{*}\left(w \mid \operatorname{TH}\left(\mathbb{A}_{\bar{E}}, \bar{E}, \operatorname{Schur}\left(\mathcal{C}_{V}\right)\right)\right)$.

\section{HOFFMAn Bounds}

The chromatic number of a graph $G=(V, E)$, denoted by $\chi(G)$, is the size of a smallest partition of $G$ into stable sets. Hoffman [26] proved the following classical lower bound on $\chi(G)$ :

$$
\chi(G) \geq 1-\frac{\lambda_{\max }\left(A_{G}\right)}{\lambda_{\min }\left(A_{G}\right)} .
$$

Here, $A_{G}$ denotes the adjacency matrix of $G$. Lovász [32 proved that the lower bound (8.1) on $\chi(G)$ remains valid if the adjacency matrix $A_{G}$ is replaced with any matrix in $\mathbb{A}_{E} \frac{\perp}{E}$, and that the tightest lower bound on $\chi(G)$ arising in this manner is precisely $\vartheta(\bar{G})$. Knuth [30, Sec. 33] defined another graph parameter, that he denoted by $\vartheta_{6}(G ; w)$, which is in fact equal to $\vartheta(G ; w)$. The parameter $\vartheta_{6}(G ; w)$ is defined as an optimization problem, and the objective function corresponding to $\vartheta_{6}(\bar{G} ; \mathbb{1})$ yields precisely the expression in the RHS of (8.1) when applied to an arbitrary matrix $A \in \mathbb{A}_{E}^{\perp}, E$. We shall extend our framework in this direction.

Let $\mathbb{A} \subseteq \mathbb{S}^{V}$ and $\mathbb{K} \subseteq \mathbb{S}^{V}$. Following Knuth [30, Sec. 33], we define

$$
\vartheta_{6}(\mathbb{A}, \mathbb{K} ; w):=\sup \left\{\lambda_{\max }(B): \operatorname{diag}(B)=w, B \in \mathbb{K}, B \in \mathbb{A}\right\}
$$

for every $w \in \mathbb{R}_{+}^{V}$. Note that the optimization problem on the RHS above is not convex. The next result relates the formulations for $\vartheta_{6}(\mathbb{A}, \mathbb{K} ; w)$ and $\vartheta_{3}(\mathbb{A}, \mathbb{K} ; w)$.

Theorem 19. Let $\mathbb{M} \subseteq \mathbb{S}^{V}$ be a diagonally scaling-invariant closed convex cone such that (4.2) holds, and that $\operatorname{Diag}\left(\mathbb{R}_{+}^{V}\right) \subseteq \mathbb{M}$. Suppose that either $\mathcal{D}_{h}(\mathbb{M}) \subseteq \mathbb{M}$ for every $h \in \mathbb{R}^{V}$ or $\mathbb{M} \subseteq \mathbb{S}_{\geq 0}^{V}$. Let $w \in \mathbb{R}_{+}^{V}$. Then

$$
\max \left\{\lambda_{\max }(B): B \in \mathbb{M}, \operatorname{diag}(B)=w\right\}=\max \left\{\sqrt{w}^{\top} X \sqrt{w}: \operatorname{Tr}(X)=1, X \in \mathbb{M}\right\} .
$$

Moreover, both optimization problems have optimal solutions. 
Proof. Equation (8.3) when $w=0$ follows from (4.2b). Thus, we may assume that $w \neq 0$. Then the RHS of (8.3) is positive, whence Lemma 4 may be applied. We start by proving ' $\geq$ ' in (8.3). Let $X^{*}$ be an optimal solution for the RHS of (8.3). Define $d$ and $\bar{X}$ as in the statement of Lemma 4 , Then $\bar{B}:=\mathcal{D}_{\sqrt{w}}(\bar{X})+\operatorname{Diag}\left(w \odot \mathbb{1}_{V \backslash \operatorname{supp}(d)}\right)$ is feasible for the LHS and its objective value is $\lambda_{\max }(\bar{B}) \geq$ $\lambda_{\max }\left(\mathcal{D}_{\sqrt{w}}(\bar{X})\right)=\sqrt{w}^{\top} X^{*} \sqrt{w}$ by (4.4C) .

Next we prove ' $\leq$ ' in (8.3). Let $\bar{B}$ be an optimal solution for the LHS of (8.3); one exists by compactness, as a consequence of (4.2c). Let $\lambda:=\lambda_{\max }(\bar{B})>0$ and let $b \in \mathbb{R}^{V}$ be a unit vector such that $\bar{B} b=\lambda b$. Note that $\operatorname{supp}(b) \subseteq \operatorname{supp}(w)$ by (4.2b). The matrix $\tilde{X}:=\operatorname{Diag}(\sqrt{w})^{\dagger} \bar{B} \operatorname{Diag}(\sqrt{w})^{\dagger} \operatorname{satisfies} \operatorname{diag}(\tilde{X})=\mathbb{1}_{\operatorname{supp}(w)}$, whence $\bar{X}:=\mathcal{D}_{b}(\tilde{X})$ satisfies $\operatorname{Tr}(\bar{X})=\|b\|^{2}=1$. If $\mathcal{D}_{h}(\mathbb{M}) \subseteq \mathbb{M}$ for each $h \in \mathbb{R}^{V}$, then $\bar{X} \in \mathbb{M}$ follows from $\bar{B} \in \mathbb{M}$. If $\mathbb{M} \subseteq \mathbb{S}_{\geq 0}^{V}$, then $\bar{X} \in \mathbb{M}$ follows from $\bar{B} \in \mathbb{M}$ and by the diagonal scaling invariance of $\mathbb{M}$, since we may assume that $b \geq 0$ by the Perron-Frobenius Theorem; see, e.g., [27, Theorem 8.3.1]. In either case, we find that $\bar{X} \in \mathbb{M}$, whence $\bar{X}$ is feasible in the RHS of (8.3). Finally, its objective value in the RHS of (8.3) is $\sqrt{w}^{\top} \bar{X} \sqrt{w}=\sqrt{w}^{\top} \mathcal{D}_{b}(\tilde{X}) \sqrt{w}=b^{\top} \mathcal{D}_{\sqrt{w}}(\tilde{X}) b=b^{\top} \bar{B} b=\lambda$, where we used (4.2b) to get $\bar{B}=\mathcal{D}_{\sqrt{w}}(\tilde{X})$. This completes the proof of (8.3).

Corollary 20. Let $\mathbb{A} \subseteq \mathbb{S}^{V}$ be a diagonally scaling-invariant polyhedral cone such that $\operatorname{Im}(\operatorname{Diag}) \subseteq \mathbb{A}$. Let $\mathbb{K} \subseteq \mathbb{S}_{+}^{V}$ be a diagonally scaling-invariant closed convex cone such that $\operatorname{Diag}\left(\mathbb{R}_{+}^{V}\right) \subseteq \mathbb{K}$. Let $w \in \mathbb{R}_{+}^{V}$. If either $\mathcal{D}_{h}(\mathbb{A} \cap \mathbb{K}) \subseteq \mathbb{A} \cap \mathbb{K}$ for all $h \in \mathbb{R}^{V}$ or $\mathbb{A} \cap \mathbb{K} \subseteq \mathbb{S}_{\geq 0}^{V}$, then

$$
\vartheta_{6}(\mathbb{A}, \mathbb{K} ; w)=\vartheta_{3}(\mathbb{A}, \mathbb{K} ; w)
$$

Proof. Immediate from Theorem 19 ,

Next we shall show that, when applied to $w=\mathbb{1}$, the objective value of the LHS of (8.3) has the same form as the RHS of (8.1), and thus generalizes it:

Proposition 21. Let $\mathbb{A}, \mathbb{K} \subseteq \mathbb{S}^{V}$ be diagonally scaling-invariant closed convex cones. Suppose that $\mathbb{A}$ is polyhedral and that $I \in \mathbb{A} \cap \mathbb{K}$. Then

$$
\vartheta_{6}(\mathbb{A}, \mathbb{K} ; \mathbb{1})=\max \left\{1-[\mu \neq 0] \frac{\lambda_{\max }(A)}{\mu}: A \in \mathbb{A} \cap \operatorname{Null}(\operatorname{diag}), \mu \in-\mathbb{R}_{+}, A \succeq_{\mathbb{K}} \mu I\right\} .
$$

Proof. We have

$$
\begin{aligned}
\max \left\{\lambda_{\max }(B): \operatorname{diag}(B)=\mathbb{1}, B \in \mathbb{A}, B \in \mathbb{K}\right\} \\
\quad=\max \left\{\lambda_{\max }(I+A): \operatorname{diag}(A)=0, I+A \in \mathbb{A}, I+A \in \mathbb{K}\right\} \\
=\max \left\{1+[\nu \neq 0] \nu \lambda_{\max }(A): A \in \mathbb{A} \cap \operatorname{Null}(\operatorname{diag}), \nu \in \mathbb{R}_{+}, \nu A \succeq_{\mathbb{K}}-I\right\} \\
\quad=\max \left\{1-[\mu \neq 0] \frac{\lambda_{\max }(A)}{\mu}: A \in \mathbb{A} \cap \operatorname{Null}(\operatorname{diag}), \mu \in-\mathbb{R}_{+}, A \succeq_{\mathbb{K}} \mu I\right\} .
\end{aligned}
$$

Note that we used Proposition 3 on the second equation. That equation also uses $I \in \mathbb{A}$, whereas the third one makes use of $I \in \mathbb{K}$.

Corollary 22. Let $G=(V, E)$ be graph. Then

$$
\begin{aligned}
& \vartheta(\bar{G} ; \mathbb{1})=\max \left\{1-[A \neq 0] \frac{\lambda_{\max }(A)}{\lambda_{\min }(A)}: A \in \mathbb{A}_{E, E}^{*}\right\}, \\
& \vartheta^{\prime}(\bar{G} ; \mathbb{1})=\max \left\{1-[A \neq 0] \frac{\lambda_{\max }(A)}{\lambda_{\min }(A)}: A \in \mathbb{A}_{E \cup \bar{E}, E}^{*}\right\}, \\
& \alpha(\bar{G} ; \mathbb{1})=\max \left\{1-[\mu \neq 0] \frac{\lambda_{\max }(A)}{\mu}: A \in \mathbb{A}_{E, E}^{*}, \mu \in-\mathbb{R}_{+}, A \succeq_{\mathcal{C}_{V}^{*}} \mu I\right\} .
\end{aligned}
$$

Moreover, all the optimization problems in (8.6) have optimal solutions.

Proof. Immediate from Theorem 13, Corollary 20, and Propositions 21 and 15. 
Finally, note that, for a graph $G=(V, E)$, we have

$$
\begin{array}{rlrl}
\max \left\{1-[A \neq 0] \frac{\lambda_{\max }(A)}{\lambda_{\min }(A)}: A \in \mathbb{A}_{E, E}^{*}\right\} & =\delta^{*}\left(\mathbb{1} \mid \operatorname{TH}\left(\mathbb{A}_{\bar{E}, \bar{E}}, \operatorname{Schur}\left(\mathbb{S}_{+}^{V}\right)\right)\right) & & \text { by Corollary 22, } \\
& \leq \delta^{*}\left(\mathbb{1} \mid \operatorname{TH}\left(\mathbb{A}_{\bar{E}}, \bar{E}, \operatorname{Schur}\left(\mathcal{C}_{V}\right)\right)\right) & & \text { since } \mathbb{S}_{+}^{V} \subseteq \mathcal{C}_{V}, \\
& =\delta^{*}(\mathbb{1} \mid \operatorname{QSTAB}(\bar{G})) & & \text { by Corollary 18, } \\
& =\chi^{*}(G ; \mathbb{1}) \leq \chi(G) . &
\end{array}
$$

This proves that the best bound from this family of lower bounds for $\chi(G)$ is $\vartheta(\bar{G})$, as was already shown by Lovász [32, Theorem 6]; see also [7].

\section{Theta Bodies over the Positive Semidefinite Cone}

The development of the theory makes it clear that the positive semidefinite cone plays a key role in theta bodies. For instance, $\mathbb{S}_{+}^{V}$ delineates the range of applicability of the lifting operators in several results (e.g., Theorems [5, 6] and 13) and it provides the most symmetric antiblocking relation (6.3), in the form of (1.4a). In this section, we focus on some special properties of theta bodies defined over the semidefinite cone. Clearly, the most interesting such families are the ones defined in (2.5). We shall reprove two classical results about these families of theta bodies using our unifying framework, and we conclude the section and the paper with a weighted extension of the convex characterization of Luz and Schrijver 37] to all semidefinite variants of $\vartheta$.

We start by reproving that every facet of a theta body over the semidefinite cone is defined by a clique inequality. We briefly recall some basic concepts of the facial structure of convex sets. Let $\mathscr{C} \subseteq \mathbb{E}$ be a convex set. A convex subset $\mathscr{F} \subseteq \mathscr{C}$ is a facet of $\mathscr{C}$ if $\operatorname{dim}(\mathscr{F})=\operatorname{dim}(\mathscr{C})-1$ and $\mathscr{F}=\arg \max _{x \in \mathscr{C}}\langle c, x\rangle$ for some nonzero $c \in \mathbb{E}^{*}$; in this case, we say that the facet $\mathscr{F}$ is determined by the inequality $\langle c, x\rangle \leq \delta^{*}(c \mid \mathscr{C})$.

The proof below is a slight modification of [43, Theorem 67.13]. Note how it uses the complementarity established in Proposition 7 :

Theorem 23. Let $\mathbb{A} \subseteq \mathbb{S}^{V}$ be a diagonally scaling-invariant polyhedral cone such that $\operatorname{Im}(\operatorname{Diag}) \subseteq \mathbb{A}$. Then each facet of $\operatorname{TH}\left(\mathbb{A}, \mathbb{S}_{+}^{\{0\} \cup V}\right)$ is determined either by $x_{i} \geq 0$ for some $i \in V$, or by $\langle w, x\rangle \leq 1$ for some $w \in \operatorname{TH}\left(\overline{\mathbb{A}}, \mathbb{S}_{+}^{\{0\} \cup V}\right) \cap\{0,1\}^{V}$.

Proof. By Corollary 2, $\mathrm{TH}\left(\mathbb{A}, \mathbb{S}_{+}^{\{0\} \cup V}\right)$ is a convex corner, and its antiblocker is $\operatorname{TH}\left(\overline{\mathbb{A}}, \mathbb{S}_{+}^{\{0\} \cup V}\right)$ by Theorem 13. By a well-known dual characterization of facets of convex corners (see, e.g., [10, Theorem 8]), it suffices to show that, if the inequality $\langle w, x\rangle \leq 1$ determines a facet of $\operatorname{TH}\left(\mathbb{A}, \mathbb{S}_{+}^{\{0\} \cup V}\right)$ for some $w \in$ $\operatorname{TH}\left(\overline{\mathbb{A}}, \mathbb{S}_{+}^{\{0\} \cup V}\right)$, then $w \in\{0,1\}^{V}$.

So let $w \in \operatorname{TH}\left(\overline{\mathbb{A}}, \mathbb{S}_{+}^{\{0\} \cup V}\right)$ such that $\langle w, x\rangle \leq 1$ determines a facet $\mathscr{F}$ of $\operatorname{TH}\left(\mathbb{A}, \mathbb{S}_{+}^{\{0\} \cup V}\right)$, and let $\hat{W} \in$ $\widehat{\mathrm{TH}}\left(\overline{\mathbb{A}}, \mathbb{S}_{+}^{\{0\} \cup V}\right)$ such that $w=\operatorname{diag}(W)$ for $W:=\hat{W}[V]$. Write $\hat{W}=\sum_{i=1}^{r}\left(\alpha_{i} \oplus a_{i}\right)\left(\alpha_{i} \oplus a_{i}\right)^{\top}$ for linearly independent vectors $\left\{\alpha_{i} \oplus a_{i}: i \in[r]\right\} \subseteq \mathbb{R}^{\{0\} \cup V}$, where $[r]:=\{1, \ldots, r\}$. Let us show that,

$$
\text { if } x \in \mathscr{F} \text {, then }\left\langle a_{i}, x\right\rangle=\alpha_{i} \text { for each } i \in[r] .
$$

So let $x \in \mathscr{F}$ and let $\hat{X} \in \widehat{\mathrm{TH}}\left(\mathbb{A}, \mathbb{S}_{+}^{\{0\} \cup V}\right)$ such that $x=\operatorname{diag}(X)$ for $X:=\hat{X}[V]$. Write $W=\operatorname{Diag}(w)-B$ for some $B \in \mathbb{A}^{*}$. Since $\hat{W} \in \widehat{\mathrm{TH}}\left(\widehat{\mathbb{A}}, \mathbb{S}_{+}^{\{0\} \cup V}\right)$ and $\hat{X} \in \widehat{\mathrm{TH}}\left(\mathbb{A}, \mathbb{S}_{+}^{\{0\} \cup V}\right)$, we have

$$
\begin{aligned}
0 & \leq\left\langle W, X-x x^{\top}\right\rangle=\langle\operatorname{Diag}(w)-B, X\rangle-x^{\top} W x=\langle w, x\rangle-\langle B, X\rangle-x^{\top} W x \\
& \leq\langle w, x\rangle-\langle w, x\rangle^{2}=1-1=0 .
\end{aligned}
$$

Equality throughout implies that $x^{\top} W x=\langle w, x\rangle^{2}=1$ and that $\langle W, X\rangle=x^{\top} W x=1$. Thus,

$$
\left\langle\left[\begin{array}{cc}
1 & -x^{\top} \\
-x & X
\end{array}\right],\left[\begin{array}{cc}
1 & w^{\top} \\
w & W
\end{array}\right]\right\rangle=0
$$

In particular,

$$
\left(\alpha_{i} \oplus a_{i}\right)^{\top}\left[\begin{array}{cc}
1 & -x^{\top} \\
-x & X
\end{array}\right]\left(\alpha_{i} \oplus a_{i}\right)=0 \quad \forall i \in[r]
$$


which implies that

$$
\left[\begin{array}{cc}
1 & -x^{\top} \\
-x & X
\end{array}\right]\left(\alpha_{i} \oplus a_{i}\right)=0 \quad \forall i \in[r]
$$

and, in particular, $\left\langle a_{i}, x\right\rangle=\alpha_{i}$ for all $i \in[r]$. This proves (9.1).

Since $\mathscr{F}$ has $n$ affinely independent vectors, (9.1) implies that $r=1$, i.e., $\hat{W}$ is rank-one. Thus, $\operatorname{diag}(W)=$ $w$ and $\hat{W} \in \mathbb{S}_{+}^{\{0\} \cup V}$ imply that $w \in\{0,1\}^{V}$.

9.1. Geometric Representations from Theta Bodies. The theta bodies described in (2.5) all have the form $\operatorname{TH}\left(\mathbb{A}_{E^{+}, E^{-}}, \mathbb{S}_{+}^{\{0\} \cup V}\right)$ for some $E^{+}, E^{-} \subseteq\left(\begin{array}{c}V \\ 2\end{array}\right)$. The elements of these sets arise from certain vectors which may be regarded as geometric representations of graphs (see, e.g., [33]):

Proposition 24. Let $E^{+}, E^{-} \subseteq\left(\begin{array}{c}V \\ 2\end{array}\right)$. Then $\mathrm{TH}\left(\mathbb{A}_{E^{+}, E^{-}}, \mathbb{S}_{+}^{\{0\} \cup V}\right)$ consists of all vectors $x \in \mathbb{R}^{V}$ of the form

$$
x_{i}=\left\langle u_{0}, u_{i}\right\rangle^{2} \quad \forall i \in V
$$

for vectors $\left\{u_{i}: i \in\{0\} \cup V\right\} \subseteq \mathbb{R}^{\{0\} \cup V}$ satisfying the following properties:

$$
\begin{array}{ll}
\left\langle u_{0}, u_{i}\right\rangle \geq 0 & \forall i \in V, \\
\left\|u_{i}\right\|=1 & \forall i \in\{0\} \cup V, \\
\left\langle u_{i}, u_{j}\right\rangle \geq 0 & \forall i j \in E^{+}, \\
\left\langle u_{i}, u_{j}\right\rangle \leq 0 & \forall i j \in E^{-} .
\end{array}
$$

Proof. Denote by $\mathscr{C}$ the set of all vectors $x \in \mathbb{R}^{V}$ of the form given by (9.2) for vectors $\left\{u_{i}: i \in\{0\} \cup V\right\} \subseteq$ $\mathbb{R}^{\{0\} \cup V}$ satisfying (9.3).

We first verify that

$$
\mathscr{C} \subseteq \mathrm{TH}\left(\mathbb{A}_{E^{+}, E^{-}}, \mathbb{S}_{+}^{\{0\} \cup V}\right) .
$$

Let $\left\{u_{i}: i \in\{0\} \cup V\right\} \subseteq \mathbb{R}^{\{0\} \cup V}$ satisfy (9.3). Define $U \in \mathbb{R}^{(\{0\} \cup V) \times V}$ by setting $U e_{i}:=u_{i}$ for every $i \in V$. Next, set $Y:=U \operatorname{Diag}\left(U^{\top} u_{0}\right)$ and

$$
\hat{X}:=\left[\begin{array}{cc}
1 & x^{\top} \\
x & X
\end{array}\right]:=\left[\begin{array}{cc}
u_{0}^{\top} u_{0} & u_{0}^{\top} Y \\
Y^{\top} u_{0} & Y^{\top} Y
\end{array}\right]=\left[\begin{array}{c}
u_{0}^{\top} \\
Y^{\top}
\end{array}\right]\left[\begin{array}{ll}
u_{0} & Y
\end{array}\right] \in \mathbb{S}_{+}^{\{0\} \cup V},
$$

where we used (9.3b). Let us verify that

$$
\begin{aligned}
X & \in \mathbb{A}_{E^{+}, E^{-}}, \\
\operatorname{diag}(X) & =x, \\
x_{i} & =\left\langle u_{0}, u_{i}\right\rangle^{2} \quad \forall i \in V .
\end{aligned}
$$

We start with (9.5a). Note that $X=Y^{\top} Y=\mathcal{D}_{U^{\top} u_{0}}\left(U^{\top} U\right)$ and $U^{\top} u_{0} \geq 0$ by (9.3a). Since $\mathbb{A}_{E^{+}, E^{-}}$is diagonally scaling-invariant, it suffices to show that $U^{\top} U \in \mathbb{A}_{E^{+}, E^{-}}$. However, this is immediate from (9.3c) and (9.3d). This proves (9.5a). For 9.5c), note that

$$
x=Y^{\top} u_{0}=\left[\operatorname{Diag}\left(U^{\top} u_{0}\right) U^{\top}\right] u_{0}=\left(U^{\top} u_{0}\right) \odot\left(U^{\top} u_{0}\right) .
$$

By (9.3b), we have $\operatorname{diag}\left(U^{\top} U\right)=\mathbb{1}$. Thus, $\operatorname{diag}(X)=\operatorname{diag}\left(\mathcal{D}_{U^{\top} u_{0}}\left(U^{\top} U\right)\right)=\left(U^{\top} u_{0}\right) \odot \operatorname{diag}\left(U^{\top} U\right) \odot\left(U^{\top} u_{0}\right)=$ $\left(U^{\top} u_{0}\right) \odot\left(U^{\top} u_{0}\right)=x$ by (9.6) , thus proving (9.5b). It follows that $x \in \mathrm{TH}\left(\mathbb{A}_{E^{+}, E^{-}}, \mathbb{S}_{+}^{\{0\} \cup V}\right)$, and the proof of (9.4) is complete.

Now we show that

$$
\operatorname{TH}\left(\mathbb{A}_{E^{+}, E^{-}}, \mathbb{S}_{+}^{\{0\} \cup V}\right) \subseteq \mathscr{C} .
$$

Let $x \in \mathrm{TH}\left(\mathbb{A}_{E^{+}, E^{-}}, \mathbb{S}_{+}^{\{0\} \cup V}\right)$, and let $\hat{X} \in \widehat{\mathrm{TH}}\left(\mathbb{A}_{E^{+}, E^{-}}, \mathbb{S}_{+}^{\{0\} \cup V}\right)$ such that $x=\operatorname{diag}(X)$ for $X:=\hat{X}[V]$. Let $Y \in \mathbb{R}^{(\{0\} \cup V) \times(\{0\} \cup V)}$ such that $\hat{X}=Y^{\top} Y$. Set $y_{i}:=Y e_{i}$ for each $i \in\{0\} \cup V$. Let $Z:=\left\{i \in V: y_{i}=0\right\}$. Define $u_{i}:=y_{i} /\left\|y_{i}\right\|$ for each $i \in\{0\} \cup(V \backslash Z)$ and let $\left\{u_{i}: i \in Z\right\}$ be an orthonormal basis for a subspace of $\left\{u_{i}: i \in\{0\} \cup(V \backslash Z)\right\}^{\perp}$ of appropriate dimension.

We must show that (9.3) holds. Note that (9.3a) for $i \in V \backslash Z$ follows from $\hat{X} e_{0}=\operatorname{diag}(\hat{X}) \geq 0$, and for $i \in Z$ it holds by construction. We also know that (9.3b) holds by construction. Let us check (9.3c). Let $i j \in E^{+}$. If $i$ or $j$ is in $Z$, then $\left\langle u_{i}, u_{j}\right\rangle=0$, so we may assume that $i, j \in V \backslash Z$. Then $\left\|y_{i}\right\|\left\|y_{j}\right\|\left\langle u_{i}, u_{j}\right\rangle=$ 
$\left\langle y_{i}, y_{j}\right\rangle=X_{i j} \geq 0$ since $X \in \mathbb{A}_{E^{+}, E^{-}}$. This completes the proof of (9.3c). The proof of (9.3d) is analogous, so (9.3) holds.

Lastly, we show that $x$ is given by (9.2). Let $i \in V$. Since $\hat{X} e_{0}=\operatorname{diag}(\hat{X})$, we have $x_{i}=\left[Y^{\top} Y\right]_{0 i}=$ $\left\langle y_{0}, y_{i}\right\rangle=\left\|y_{0}\right\|\left\|y_{i}\right\|\left\langle u_{0}, u_{i}\right\rangle=X_{i i}^{1 / 2}\left\langle u_{0}, u_{i}\right\rangle=x_{i}^{1 / 2}\left\langle u_{0}, u_{i}\right\rangle$. If $x_{i}>0$, then $x_{i}^{1 / 2}=\left\langle u_{0}, u_{i}\right\rangle$. Otherwise, $u_{i} \perp u_{0}$ by construction, so $x_{i}=0=\left\langle u_{0}, u_{i}\right\rangle^{2}$. This proves that $x$ is given by (9.2) and completes the proof of (9.7).

An orthonormal representation of a graph $G=(V, E)$ is a map $u$ that sends $V$ into the unit vectors of some Euclidean space such that $\left\langle u_{i}, u_{j}\right\rangle=0$ whenever $i j \in \bar{E}$. If, additionally, $\left\langle u_{i}, u_{j}\right\rangle \geq 0$ whenever $i j \in E$, then $u$ is called an acute orthonormal representation of $G$. Finally, an obtuse representation of $G$ is a map $u$ from $V$ to the unit vectors of some Euclidean space so that $\left\langle u_{i}, u_{j}\right\rangle \leq 0$ whenever $i j \in \bar{E}$.

Proposition 24 immediately leads to the following well-known internal description of the sets in (2.5).

Corollary 25. Let $G=(V, E)$ be a graph. Let $\mathscr{C} \in\left\{\mathrm{TH}(G), \mathrm{TH}^{\prime}(G), \mathrm{TH}^{+}(G)\right\}$. Then $\mathscr{C}$ consists of all vectors $x \in \mathbb{R}^{V}$ of the form $x_{i}=\left\langle u_{0}, u_{i}\right\rangle^{2}$ for every $i \in V$ for some unit vectors in $\left\{u_{i}: i \in\{0\} \cup V\right\} \subseteq \mathbb{R}^{\{0\} \cup V}$ such that

(i) $u \uparrow_{V}$ is an orthonormal representation of $\bar{G}$, if $\mathscr{C}=\mathrm{TH}(G)$;

(ii) $u \uparrow_{V}$ is an acute orthonormal representation of $\bar{G}$ and $\left\langle u_{0}, u_{i}\right\rangle \geq 0$ for all $i \in V$, if $\mathscr{C}=\mathrm{TH}^{\prime}(G)$;

(iii) $u \uparrow_{V}$ is an obtuse representation of $\bar{G}$ and $\left\langle u_{0}, u_{i}\right\rangle \geq 0$ for all $i \in V$, if $\mathscr{C}=\mathrm{TH}^{+}(G)$.

Proof. Immediate from Proposition 24. When $\mathscr{C}=\mathrm{TH}(G)$, the constraint $\left\langle u_{0}, u_{i}\right\rangle \geq 0$ may be dropped, since for each orthonormal representation $u$ of $G$ and $i \in V$, the map obtained from $u$ by replacing some image $u_{i}$ by $-u_{i}$ is also an orthonormal representation of $G$.

9.2. Luz and Schrijver's Convex Quadratic Characterization. In this subsection, we show how to generalize the convex quadratic characterization from [37] to the context of generalized theta bodies over the cone $\widehat{\mathbb{K}}=\mathbb{S}_{+}^{\{0\} \cup V}$. This provides a generalization of their results to all weights $w \in \mathbb{R}_{+}^{V}$ to all of the functions $\vartheta, \vartheta^{\prime}$, and $\vartheta^{+}$. We remark that a convex quadratic characterization of $\vartheta^{\prime}$ was already known to Luz [35, as well as a weighted generalization of the convex quadratic characterization of $\vartheta$, which appeared in an unpublished report (in Portuguese) by Luz in 2005 [36.

Let $C \in \mathbb{S}^{V}$ such that $\operatorname{diag}(C)=0$. For each $w \in \mathbb{R}_{+}^{V}$, define

$$
v(C ; w):=\max \left\{2\langle w, x\rangle-x^{\top} \mathcal{D}_{\sqrt{w}}\left(H_{C}+I\right) x: x \in \mathbb{R}_{+}^{V}\right\},
$$

where

$$
H_{C}:=[C \neq 0] \frac{C}{-\lambda_{\min }(C)} .
$$

Note that $\operatorname{diag}(C)=0$ implies that $H_{C}+I \succeq 0$, so the quadratic program on the RHS of (9.8) is convex. In particular, there is an optimal solution whenever the optimal value is finite. The necessary and sufficient conditions for optimality are:

$$
\begin{gathered}
x \geq 0, \\
\mathcal{D}_{\sqrt{w}}\left(H_{C}+I\right) x \geq w, \\
x^{\top} \mathcal{D}_{\sqrt{w}}\left(H_{C}+I\right) x=\langle w, x\rangle=v(C ; w) .
\end{gathered}
$$

Let $\mathbb{A} \subseteq \mathbb{S}^{V}$ be a polyhedral diagonally scaling-invariant cone. Define

$$
v(\mathbb{A} ; w):=\inf \{v(C ; w): C \in \mathbb{A} \cap \operatorname{Null}(\operatorname{diag})\} .
$$

We first show that $v(\mathbb{A} ; w)$ provides an upper bound for $\vartheta_{1}\left(\overline{\mathbb{A}}, \mathbb{S}_{+}^{V}\right)$. Note the similarity with the proof of Theorem 5 .

Proposition 26. Let $\mathbb{A} \subseteq \mathbb{S}^{V}$ be a diagonally scaling-invariant closed convex cone such that $\operatorname{Im}(\operatorname{Diag}) \subseteq \mathbb{A}$. Let $w \in \mathbb{R}_{+}^{V}$. Then

$$
\min \left\{\max _{i \in V} \frac{w_{i}}{y_{i}}: y \in \mathrm{TH}\left(\mathbb{A}, \mathbb{S}_{+}^{\{0\} \cup V}\right)\right\} \leq v(\mathbb{A} ; w)
$$


Proof. Let $C \in \mathbb{A} \cap \operatorname{Null(diag)~such~that~} v:=v(C ; w)<\infty$, and let $\bar{x}$ be an optimal solution for the corresponding optimization problem (9.8). We shall use the optimality conditions (9.10) without further mention. We may assume that $\operatorname{supp}(\bar{x}) \subseteq \operatorname{supp}(w)$, so we may also assume that $\operatorname{supp}(w)=V$ and that $v>0$. Write $H_{C}+I \succeq 0$ as $H_{C}+I=B^{\top} B$ for some $B \in \mathbb{R}^{V \times V}$. Set

$$
\begin{gathered}
c:=v^{-1 / 2} B \operatorname{Diag}(\sqrt{w}) \bar{x}, \\
z:=B^{\top} c, \\
\bar{B}:=B \operatorname{Diag}\left(B^{\top} c\right), \\
y:=\bar{B}^{\top} c=\operatorname{Diag}(z) z=z \odot z .
\end{gathered}
$$

Note that $\mathcal{D}_{\sqrt{w}}\left(H_{C}+I\right) \bar{x} \geq w$ implies that

$$
z=v^{-1 / 2}\left(H_{C}+I\right) \operatorname{Diag}(\sqrt{w}) \bar{x} \geq v^{-1 / 2} \sqrt{w} .
$$

We claim that

$$
\hat{Y}:=\left[\begin{array}{cc}
1 & y^{\top} \\
y & Y
\end{array}\right]:=\left[\begin{array}{cc}
1 & y^{\top} \\
y & \bar{B}^{\top} \bar{B}
\end{array}\right] \in \operatorname{TH}\left(\mathbb{A}, \mathbb{S}_{+}^{\{0\} \cup V}\right) .
$$

Positive semidefiniteness of the matrix in (9.14) follows from the factorization

$$
\left[\begin{array}{cc}
1 & y^{\top} \\
y & \bar{B}^{\top} \bar{B}
\end{array}\right]=\left[\begin{array}{c}
c^{\top} \\
\bar{B}^{\top}
\end{array}\right]\left[\begin{array}{ll}
c & \bar{B}
\end{array}\right]
$$

Since we have $H_{C}+I \in \mathbb{A}$ and $z \geq 0$ follows from (9.13), we get $\bar{B}^{\top} \bar{B}=\mathcal{D}_{z}\left(H_{C}+I\right) \in \mathbb{A}$. Finally

$$
\operatorname{diag}\left(\bar{B}^{\top} \bar{B}\right)=z \odot \operatorname{diag}\left(B^{\top} B\right) \odot z=y
$$

since $\operatorname{diag}(C)=0$. This concludes the proof of (9.14), and so we have $y \in \operatorname{TH}\left(\mathbb{A}, \mathbb{S}_{+}^{\{0\} \cup V}\right)$. It follows from (9.13) that $y_{i} \geq v^{-1} w_{i}$ for each $i \in V$, so that $\max _{i \in V} w_{i} / y_{i} \leq v$.

Next we show how $v(\mathbb{A} ; w)$ relates to $\vartheta_{2}\left(\overline{\mathbb{A}}, \mathbb{S}_{+}^{V}\right)$ :

Theorem 27. Let $\mathbb{A} \subseteq \mathbb{S}^{V}$ be a polyhedral diagonally scaling-invariant cone such that $\operatorname{Im}(\operatorname{Diag}) \subseteq \mathbb{A}$. Let $w \in \mathbb{R}_{+}^{V}$. Let $\bar{Y}$ be an optimal solution for

$$
\vartheta:=\min \left\{\lambda_{\max }\left(Y+\sqrt{w} \sqrt{w}^{\top}\right): Y \in-\mathbb{A} \cap \operatorname{Null}(\operatorname{diag})\right\} .
$$

Then $\vartheta \geq v(-\bar{Y} ; w)$.

Proof. First note that the dual of (9.15) has $\frac{1}{|V|} I$ as a restricted Slater point, so an optimal solution for (9.15) exists and $\vartheta \geq 0$, with equality only if $w=0$. We may thus assume that $\vartheta>0$. Set $\bar{C}:=-\bar{Y}$. Clearly, $\vartheta \geq \lambda_{\max }(\bar{Y})$. If $\vartheta=\lambda_{\max }(\bar{Y})$, then the objective value of an arbitrary $x \in \mathbb{R}_{+}^{V}$ in the formulation for $v(C ; w)$ is

$$
\begin{aligned}
2\langle w, x\rangle-x^{\top} \mathcal{D}_{\sqrt{w}}\left(H_{\bar{C}}+I\right) x & =2\langle w, x\rangle-\vartheta^{-1} x^{\top} \mathcal{D}_{\sqrt{w}}\left(\vartheta I-\bar{Y}-\sqrt{w} \sqrt{w}^{\top}\right) x-\vartheta^{-1}\langle w, x\rangle^{2} \\
& \leq 2\langle w, x\rangle-\vartheta^{-1}\langle w, x\rangle^{2} \leq \vartheta
\end{aligned}
$$

since $\left(\vartheta^{1 / 2}-\vartheta^{-1 / 2}\langle w, x\rangle\right)^{2} \geq 0$, and hence $v(-\bar{Y} ; w) \leq \vartheta$. Thus, we may assume that

$$
\vartheta>\lambda_{\max }(\bar{Y}) \text {. }
$$

Let $\bar{B}$ be an optimal solution for the dual of (9.15), given by $\sup \left\{\sqrt{w}^{\top} B \sqrt{w}: \operatorname{Tr}(B)=1, B \in \overline{\mathbb{A}} \cap \mathbb{S}_{+}^{V}\right\}$. Note that $\vartheta I-\bar{Y}$ is nonsingular by (9.16), so the rank of $\vartheta I-\bar{Y}-\sqrt{w} \sqrt{w}^{\top}$ is $\geq|V|-1$. Since $\langle\bar{B}, \vartheta I-$ $\left.\bar{Y}-\sqrt{w} \sqrt{w}^{\top}\right\rangle=0$ by complementarity, it follows that $\bar{B}$ has rank one. Write $\bar{B}=\vartheta^{-1} \bar{y} \bar{y}^{\top}$ with $\bar{y}$ having at least one positive component, set $S:=\operatorname{supp}(\bar{y})$ and $\bar{x}:=\mathbb{1}_{S}$. Let us show that

$$
\begin{gathered}
\bar{y}=\operatorname{Diag}(\sqrt{w}) \bar{x}, \\
S \subseteq \operatorname{supp}(w), \\
\langle\sqrt{w}, \bar{y}\rangle=\langle w, \bar{x}\rangle=\vartheta, \\
\|y\|^{2}=\vartheta .
\end{gathered}
$$


We shall apply Lemma 4 The inclusion (9.17b) follows from (4.4a). Equation (4.4d yields $\langle\sqrt{w}, \bar{y}\rangle \bar{y}=$ $\vartheta \operatorname{Diag}(\sqrt{w})^{\dagger} \operatorname{Diag}(\bar{y}) \bar{y}$. If we multiply this equation on the left by $\operatorname{Diag}(\sqrt{w}) \operatorname{Diag}(\bar{y})^{\dagger}$ and use (9.17b) we get

$$
\langle\sqrt{w}, \bar{y}\rangle \operatorname{Diag}(\sqrt{w}) \bar{x}=\vartheta \bar{y} .
$$

Thus, $\bar{y} \geq 0$ or $\bar{y} \leq 0$, so that $\bar{y} \geq 0$ since $\bar{y}$ has at least one positive component. Then $\vartheta=\sqrt{w}^{\top} \bar{B} \sqrt{w}$ implies that $\langle\sqrt{w}, \bar{y}\rangle=\vartheta$, which establishes (9.17a) via (9.18) and half of (9.17c); the other half follows from the half already established and (9.17a). Finally, (9.17d) follows from $\operatorname{Tr}(\bar{B})=1$.

We claim that

$$
\bar{x} \text { satisfies the optimality conditions (9.10) for } \vartheta=v(\bar{C} ; w) \text {. }
$$

Clearly (9.10a) holds. Since complementarity yields $\left(\vartheta I-\bar{Y}-\sqrt{w} \sqrt{w}^{\top}\right) \bar{y}=0$, we have $\vartheta \bar{y}=\left(\sqrt{w} \sqrt{w}^{\top}+\bar{Y}\right) \bar{y}$ and thus $-\bar{Y} \bar{y}=\vartheta(\sqrt{w}-\bar{y})$ using (9.17c). Thus,

$$
\mathcal{D}_{\sqrt{w}}\left(H_{\bar{C}}+I\right) \bar{x}=\operatorname{Diag}(\sqrt{w})\left[\frac{-\bar{Y}}{\lambda_{\max }(\bar{Y})}+I\right] \bar{y}=\operatorname{Diag}(\sqrt{w})\left[\frac{\vartheta}{\lambda_{\max }(\bar{Y})}(\sqrt{w}-\bar{y})+\bar{y}\right] .
$$

Since $\vartheta \geq \lambda_{\max }(\bar{Y})$ and $\sqrt{w} \geq \bar{y}$, we get from (9.20) that $\left.\mathcal{D}_{\sqrt{w}}\left(H_{\bar{C}}+I\right) \bar{x} \geq \operatorname{Diag}(\sqrt{w})[(\sqrt{w}-\bar{y})+\bar{y}]\right)=w$, so (9.10b) holds. By hitting the LHS of (9.20) on the left with $\bar{x}^{\mathrm{T}}$, we get from the RHS using (9.17) that $\bar{x}^{\top} \mathcal{D}_{\sqrt{w}}\left(H_{\bar{C}}+I\right) \bar{x}=\vartheta$. Thus, (9.10c) follows from (9.17c), and the proof of (9.19) is complete.

Corollary 28. Let $\mathbb{A} \subseteq \mathbb{S}^{V}$ be a polyhedral diagonally scaling-invariant cone such that $\operatorname{Im}(\operatorname{Diag}) \subseteq \mathbb{A}$. Let $w \in \mathbb{R}_{+}^{V}$. Then

$$
v(\mathbb{A} ; w)=\vartheta\left(\overline{\mathbb{A}}, \mathbb{S}_{+}^{V} ; w\right) .
$$

Moreover, the optimization problem for $v(\mathbb{A} ; w)$ has an optimal solution.

Proof. Immediate from Proposition 26 and Theorem 27

\section{CONCLUSION}

We took an axiomatic viewpoint in our study of the Lovász theta function and the related theta body of graphs. We generalized the binary encoding of graphs by the notion of diagonally scaling-invariant polyhedral cones and the semidefinite cone with a more general set of convex cones. These generalization and viewpoint led to graph complementation being replaced by convex polarity and to the new notion of Schur-Lifting of cones as the dual of commonly used PSD-Lifting of cones. Our new general theory has many advantages: we are able to treat the stable set polytope and many of its convex relaxations uniformly and extend the most commonly used equivalent characterizations of Lovász theta function and the most powerful properties to these generalized theta bodies.

\section{REFERENCES}

[1] N. E. Aguilera, M. S. Escalante, and G. L. Nasini. A generalization of the perfect graph theorem under the disjunctive index. Math. Oper. Res., 27(3):460-469, 2002.

[2] Noga Alon, Konstantin Makarychev, Yury Makarychev, and Assaf Naor. Quadratic forms on graphs. Invent. Math., 163(3):499-522, 2006.

[3] C. Bachoc, G. Nebe, F. M. de Oliveira Filho, and F. Vallentin. Lower bounds for measurable chromatic numbers. Geom. Funct. Anal., 19(3):645-661, 2009.

[4] R. Bačík. Structure of Graph Homomorphisms. PhD thesis, Simon Fraser University, 1997.

[5] R. Bačík and S. Mahajan. Semidefinite programming and its applications to NP problems. In Computing and combinatorics (Xi'an, 1995), volume 959 of Lecture Notes in Comput. Sci., pages 566-575. Springer, Berlin, 1995.

[6] A. Ben-Tal and A. Nemirovski. Lectures on modern convex optimization. MPS/SIAM Series on Optimization. Society for Industrial and Applied Mathematics (SIAM), Philadelphia, PA; Mathematical Programming Society (MPS), Philadelphia, PA, 2001. Analysis, algorithms, and engineering applications.

[7] Y. Bilu. Tales of Hoffman: three extensions of Hoffman's bound on the graph chromatic number. J. Combin. Theory Ser. $B, 96(4): 608-613,2006$.

[8] M. K. de Carli Silva. Geometric Ramifications of the Lovász Theta Function and Their Interplay with Duality. PhD thesis, Department of Combinatorics and Optimization, University of Waterloo, 2013. http://hdl.handle.net/10012/7812

[9] M. K. de Carli Silva and L. Tunçel. Optimization problems over unit-distance representations of graphs. Electron. J. Combin., 20(1):Research Paper 43, 20 pp. (electronic), 2013.

[10] M. K. de Carli Silva and L. Tunçel. Vertices of spectrahedra arising from the elliptope, the theta body, and their relatives. ArXiv e-prints, September 2013. To appear in SIAM J. Optim. 
[11] Y. H. Chan and L. C. Lau. On linear and semidefinite programming relaxations for hypergraph matching. Math. Program., 135(1-2, Ser. A):123-148, 2012.

[12] I. Csiszár, J. Körner, L. Lovász, K. Marton, and G. Simonyi. Entropy splitting for antiblocking corners and perfect graphs. Combinatorica, 10(1):27-40, 1990.

[13] E. de Klerk and D. V. Pasechnik. Approximation of the stability number of a graph via copositive programming. SIAM J. Optim., 12(4):875-892 (electronic), 2002.

[14] I. Dukanovic and F. Rendl. Copositive programming motivated bounds on the stability and the chromatic numbers. Math. Program., 121(2, Ser. A):249-268, 2010.

[15] U. Feige and L. Lovász. Two-prover one-round proof systems: their power and their problems (extended abstract). In Proceedings of the twenty-fourth annual ACM symposium on Theory of computing, STOC '92, pages 733-744, New York, NY, USA, 1992. ACM.

[16] A. Galtman. Spectral characterizations of the Lovász number and the Delsarte number of a graph. J. Algebraic Combin., 12(2):131-143, 2000.

[17] A. M. H. Gerards, G. Maróti, and A. Schrijver. A note on: "A generalization of the perfect graph theorem under the disjunctive index" [Math. Oper. Res. 27 (2002), no. 3, 460-469; mr1926653] by N. E. Aguilera, M. S. Escalante and G. L. Nasini. Math. Oper. Res., 28(4):884-885, 2003.

[18] L. E. Gibbons, D. W. Hearn, P. M. Pardalos, and M. V. Ramana. Continuous characterizations of the maximum clique problem. Math. Oper. Res., 22(3):754-768, 1997.

[19] D. Gijswijt. Matrix Algebras and Semidefinite Programming Techniques for Codes. PhD thesis, University of Amsterdam, 2005.

[20] M. X. Goemans. Semidefinite programming in combinatorial optimization. Math. Programming, 79(1-3, Ser. B):143-161, 1997. Lectures on mathematical programming (ismp97) (Lausanne, 1997).

[21] J. Gouveia, P. A. Parrilo, and R. R. Thomas. Theta bodies for polynomial ideals. SIAM J. Optim., 20(4):2097-2118, 2010.

[22] M. Grötschel, L. Lovász, and A. Schrijver. Geometric algorithms and combinatorial optimization, volume 2 of Algorithms and Combinatorics: Study and Research Texts. Springer-Verlag, Berlin, 1988.

[23] M. Grötschel, L. Lovász, and A. Schrijver. Geometric algorithms and combinatorial optimization, volume 2 of Algorithms and Combinatorics. Springer-Verlag, Berlin, second edition, 1993.

[24] O. Güler. Barrier functions in interior point methods. Math. Oper. Res., 21(4):860-885, 1996.

[25] N. Gvozdenović and M. Laurent. The operator $\Psi$ for the chromatic number of a graph. SIAM J. Optim., 19(2):572-591, 2008.

[26] A. J. Hoffman. On eigenvalues and colorings of graphs. In Graph Theory and its Applications (Proc. Advanced Sem., Math. Research Center, Univ. of Wisconsin, Madison, Wis., 1969), pages 79-91. Academic Press, New York, 1970.

[27] R. A. Horn and C. R. Johnson. Matrix analysis. Cambridge University Press, Cambridge, 1990. Corrected reprint of the 1985 original.

[28] D. Karger, R. Motwani, and M. Sudan. Approximate graph coloring by semidefinite programming. J. ACM, 45(2):246-265, 1998.

[29] J. Kleinberg and M. X. Goemans. The Lovász theta function and a semidefinite programming relaxation of vertex cover. SIAM J. Discrete Math., 11(2):196-204 (electronic), 1998.

[30] D. E. Knuth. The sandwich theorem. Electron. J. Combin., 1:Article 1, approx. 48 pp. (electronic), 1994.

[31] L. Lipták and L. Tunçel. Lift-and-project ranks and antiblocker duality. Oper. Res. Lett., 33(1):35-41, 2005.

[32] L. Lovász. On the Shannon capacity of a graph. IEEE Trans. Inform. Theory, 25(1):1-7, 1979.

[33] L. Lovász. Geometric representations of graphs. http://www.cs.elte.hu/ lovasz/geomrep.pdf, 2009. Accessed: 2014-0908.

[34] L. Lovász and A. Schrijver. Cones of matrices and set-functions and 0-1 optimization. SIAM J. Optim., 1(2):166-190, 1991.

[35] C. J. Luz. A convex quadratic characterization of the McEliece-Rodemich-Rumsey and Schrijver upper bound on the stability number of a graph. Cadernos de Matemática CM06/I-14, Departamento de Matemática, Universidade de Aveiro, 2006.

[36] C. J. Luz. Private communication, November 2014.

[37] C. J. Luz and A. Schrijver. A convex quadratic characterization of the Lovász theta number. SIAM J. Discrete Math., 19(2):382-387 (electronic), 2005.

[38] K. Marton. On the Shannon capacity of probabilistic graphs. J. Combin. Theory Ser. B, 57(2):183-195, 1993.

[39] R. J. McEliece, E. R. Rodemich, and H. C. Rumsey, Jr. The Lovász bound and some generalizations. J. Combin. Inform. System Sci., 3(3):134-152, 1978.

[40] Yu. Nesterov and A. Nemirovskii. Interior-point polynomial algorithms in convex programming, volume 13 of SIAM Studies in Applied Mathematics. Society for Industrial and Applied Mathematics (SIAM), Philadelphia, PA, 1994.

[41] R. T. Rockafellar. Convex analysis. Princeton Landmarks in Mathematics. Princeton University Press, Princeton, NJ, 1997. Reprint of the 1970 original, Princeton Paperbacks.

[42] A. Schrijver. A comparison of the Delsarte and Lovász bounds. IEEE Trans. Inform. Theory, 25(4):425-429, 1979.

[43] A. Schrijver. Combinatorial optimization. Polyhedra and efficiency. Vol. B, volume 24 of Algorithms and Combinatorics. Springer-Verlag, Berlin, 2003. Matroids, trees, stable sets, Chapters 39-69.

[44] G. Simonyi. Perfect graphs and graph entropy. An updated survey. In Perfect graphs, Wiley-Intersci. Ser. Discrete Math. Optim., pages 293-328. Wiley, Chichester, 2001.

[45] M. Szegedy. A note on the theta number of Lovász and the generalized Delsarte bound. In Proceedings of the 35th Annual IEEE Symposium on Foundations of Computer Science, 1994. 
[46] É. Tardos. The gap between monotone and nonmonotone circuit complexity is exponential. Combinatorica, 8(1):141-142, 1988.

[47] V. A. Truong and L. Tunçel. Geometry of homogeneous convex cones, duality mapping, and optimal self-concordant barriers. Math. Program., 100(2):295-316, 2004.

\section{Appendix A. Antiblocking Duality}

Antiblocking duality is the form of duality most suitable for convex corners, and we used extensively the basic facts of this duality theory in this paper. Even though such facts are well known, we are not aware of any previous treatment in the literature of antiblocking duality for non-polyhedral convex corners which meets the needs of this paper. Thus, for the sake of completeness, we include a brief, self-contained description of this theory.

Recall from Section 2 that a convex corner is a compact, lower-comprehensive convex subset of the nonnegative orthant with nonempty interior, and that the antiblocker of a convex corner $\mathscr{C} \subseteq \mathbb{R}_{+}^{n}$ is abl $(\mathscr{C}):=$ $\left\{y \in \mathbb{R}_{+}^{n}:\langle y, x\rangle \leq 1 \forall x \in \mathscr{C}\right\}$. Note that $\operatorname{abl}(\mathscr{C})$ is also a convex corner. Closedness, convexity and inclusion in $\mathbb{R}_{+}^{n}$ are clear, whereas lower-comprehensiveness follows from the inclusion $\mathscr{C} \subseteq \mathbb{R}_{+}^{n}$. Boundedness of abl $(\mathscr{C})$ follows from $\mathscr{C} \cap \mathbb{R}_{++}^{n} \neq \varnothing$, since $\operatorname{int}(\mathscr{C}) \neq \varnothing$. Finally, since $\mathscr{C}$ is bounded, abl $(\mathscr{C})$ has nonempty interior: if $M \in \mathbb{R}$ is such that $x \leq n^{-1} M \mathbb{1}$ for all $x \in \mathscr{C}$, then $M^{-1} \mathbb{1} \in \operatorname{abl}(\mathscr{C})$, so that $\frac{1}{2} M^{-1} \mathbb{1} \in \operatorname{int}(\operatorname{abl}(\mathscr{C}))$ by lower-comprehensiveness of $\operatorname{abl}(\mathscr{C})$.

The key fact of antiblocking duality is that abl(.) defines an involution on the class of convex corners, which we prove next.

Theorem 29. If $\mathscr{C} \subseteq \mathbb{R}_{+}^{n}$ is a convex corner, then $\operatorname{abl}(\operatorname{abl}(\mathscr{C}))=\mathscr{C}$.

Proof. The inclusion ' $\supseteq$ ' is trivial. For the other inclusion, let $u \in \mathbb{R}^{n} \backslash \mathscr{C}$. If $u \notin \mathbb{R}_{+}^{n}$, then $u \in \mathbb{R}^{n} \backslash$ $\operatorname{abl}(\operatorname{abl}(\mathscr{C}))$, so assume that $u \geq 0$. Let $x^{*} \in \mathscr{C}$ minimize $\|u-x\|^{2}$ over $x \in \mathscr{C}$. Set

$$
y:=u-x^{*} \quad \text { and } \quad \mu:=\left\langle y, x^{*}\right\rangle \text {. }
$$

We claim that

$$
\langle y, x\rangle \leq \mu \quad \forall x \in \mathscr{C}, \quad \text { and } \quad\langle y, u\rangle>\mu .
$$

Let $x \in \mathscr{C}$. If $t \in(0,1]$, then $x^{*}+t\left(x-x^{*}\right) \in \mathscr{C}$, so that $\left\|u-x^{*}\right\|^{2} \leq\left\|u-x^{*}-t\left(x-x^{*}\right)\right\|^{2}$, whence $\left\langle u-x^{*}, x-x^{*}\right\rangle \leq t\left\|x-x^{*}\right\|^{2} / 2$. By sending $t \downarrow 0$, we get $\left\langle u-x^{*}, x-x^{*}\right\rangle \leq 0$, which implies the first half of (A.1). The second half is easily seen to be equivalent to $\|y\|^{2}>0$, which follows from the fact that $x^{*} \in \mathscr{C}$ whereas $u \notin \mathscr{C}$. This concludes the proof of (A.1).

Next we show that

$$
y \geq 0 .
$$

Suppose that $y_{i}<0$ for some $i \in\{1, \ldots, n\}$. Then $x_{i}^{*}>u_{i} \geq 0$. Let $\varepsilon>0$ such that $x_{i}^{*} \geq \varepsilon$ and set $\bar{x}:=x^{*}-\varepsilon e_{i} \in \mathscr{C}$. By (A.1), we get $\mu \geq\langle y, \bar{x}\rangle=\left\langle y, x^{*}\right\rangle-\varepsilon\left\langle y, e_{i}\right\rangle=\mu-\varepsilon y_{i}>\mu$, a contradiction. This proves (A.2), which yields $\mu>0$ when combined with (A.1) and $\operatorname{int}(\mathscr{C}) \neq \varnothing$. Now (A.1) and (A.2) show that $\frac{1}{\mu} y \in \operatorname{abl}(\mathscr{C})$, so $u \in \mathbb{R}^{n} \backslash \operatorname{abl}(\operatorname{abl}(\mathscr{C}))$ by (A.1). This concludes the proof of ' $\subseteq$ '.

We thus obtain the following optimality conditions:

Corollary 30. Let $\mathscr{C} \subseteq \mathbb{R}_{+}^{n}$ be a convex corner. Let $w \in \mathbb{R}_{+}^{n}$, and let $\vartheta \in \mathbb{R}_{+}$. Then $\delta^{*}(w \mid \operatorname{abl}(\mathscr{C}))=\vartheta$ if and only if there exists $x^{*} \in \operatorname{abl}(\mathscr{C})$ and $y^{*} \in \mathscr{C}$ such that $\left\langle y^{*}, x^{*}\right\rangle=1$ and $\vartheta y^{*}=w$.

Proof. First we prove necessity. If $w=0$, let $x^{*} \in \operatorname{abl}(\mathscr{C})$ maximize $\|x\|^{2}$ over $x \in \operatorname{abl}(\mathscr{C})$, and set $y^{*}:=x^{*} /\left\|x^{*}\right\|^{2} ;$ if $x \in \operatorname{abl}(\mathscr{C})$, then $\left\langle y^{*}, x\right\rangle \leq\|x\|\left\|x^{*}\right\| /\left\|x^{*}\right\|^{2} \leq 1$ by Cauchy-Schwarz, so $y^{*} \in \mathscr{C}$ by Theorem 29. Assume that $w \neq 0$, so $\vartheta>0$. Let $x^{*}$ be maximize $\langle w, x\rangle$ over $x \in \operatorname{abl}(\mathscr{C})$, and set $y^{*}:=w / \vartheta$. If $x \in \operatorname{abl}(\mathscr{C})$, then $\left\langle y^{*}, x\right\rangle \leq 1$ since $\vartheta=\delta^{*}(w \mid \operatorname{abl}(\mathscr{C}))$, so $y^{*} \in \mathscr{C}$ by Theorem 29.

Now we prove sufficiency. If $x \in \operatorname{abl}(\mathscr{C})$, then $\langle w, x\rangle=\vartheta\left\langle y^{*}, x\right\rangle \leq \vartheta$ shows that $\delta^{*}(w \mid \operatorname{abl}(\mathscr{C})) \leq \vartheta$, whereas $\left\langle w, x^{*}\right\rangle=\vartheta\left\langle y^{*}, x^{*}\right\rangle=\vartheta$ shows that equality holds. 